\title{
Ten years of progress in the Hokkaido birth cohort study on environment and children's health: cohort profile-updated 2013
}

\author{
Reiko Kishi - Sachiko Kobayashi · Tamiko Ikeno • Atsuko Araki • Chihiro Miyashita • \\ Sachiko Itoh · Seiko Sasaki • Emiko Okada - Sumitaka Kobayashi - Ikuko Kashino • Kumiko Itoh • \\ Sonomi Nakajima - The members of the Hokkaido Study on Environment and Children's Health
}

Received: 25 July 2013/ Accepted: 30 July 2013/Published online: 20 August 2013

(C) The Japanese Society for Hygiene 2013

\begin{abstract}
The Hokkaido Study on Environment and Children's Health is an ongoing cohort study that began in 2002. The study consists of two prospective birth cohorts, the Sapporo cohort $(n=514)$ and the Hokkaido large-scale cohort $(n=20,940)$. The primary goals of this study are to first examine the potential negative effects of perinatal environmental chemical exposures on birth outcomes, including congenital malformations and growth retardation; second, to evaluate the development of allergies, infectious diseases and neurodevelopmental disorders and perform longitudinal observations of the children's physical development to clarify the causal relationship between these outcomes and environmental chemicals; third, to identify individuals genetically susceptible to environmental chemicals; finally, to identify the additive effects of various environmental factors in our daily life, such as secondhand smoke exposure or low folate intake during early pregnancy. In this paper, we introduce our recent progress in the Hokkaido study with a cohort profile updated in 2013. For the last ten years, we followed
\end{abstract}

The members of the Hokkaido Study on Environment and Children's Health are listed in the "Appendix".

R. Kishi $(\bowtie) \cdot$ S. Kobayashi · T. Ikeno · A. Araki ·

C. Miyashita $\cdot$ S. Itoh

Hokkaido University Center for Environmental and Health

Sciences, Kita 12, Nishi 7, Kita-ku, Sapporo 060-0812, Japan

e-mail: rkishi@med.hokudai.ac.jp

S. Sasaki · E. Okada - S. Kobayashi - I. Kashino - K. Itoh Department of Public Health Sciences, Hokkaido University Graduate School of Medicine, Sapporo, Japan

S. Nakajima

Department of Occupational Therapy, School of Health

Sciences, Sapporo Medical University, Sapporo, Japan pregnant women and their offspring, measuring various environmental chemicals, i.e., $\mathrm{PCB}, \mathrm{OH}-\mathrm{PCB}$ and dioxins, PFCs (Perfluorinated Compounds), Organochlorine pesticides, Phthalates, bisphenol A and mercury. We discovered that the concentration of toxic equivalents (TEQ) of dioxin and other specific congeners of PCDF or PCDD have effects on birth weight, infants' neurodevelopment and immune function. There were significant gender differences in these effects; our results suggest that male infants have more susceptibility to those chemical exposures than female infants. Interestingly, we found maternal genetic polymorphisms in AHR, CYP1Al or GSTs that significantly modified the dioxin concentrations in maternal blood, suggesting different dioxin accumulations in the bodies of individuals with these genotypes, which would lead to different dioxin exposure levels. These genetic susceptibility factors influenced the body size of children born from mothers that either smoked or were passively exposed to tobacco smoke. Further studies investigating the correlation between epigenetics, the effects of intrauterine exposure to environmental chemicals and developmental factors related to health and disease are warranted.

Keywords Birth cohort - PCB/dioxin - PFCs (PFAAs) - Gene-environment interaction · Gender difference

\section{Introduction}

In 1997, Theo Colborn et al. [1] warned of the dangers of environmental chemicals, which act as endocrine disruptors and can eventually led to impairments in reproductive capacity. Since that warning, a myriad of animal and epidemiological studies have been conducted to evaluate the 
adverse health effects of these endocrine-disrupting chemicals (EDCs) [2-4]. Currently, these chemicals are considered to contribute to numerous adverse health effects, including growth retardation of fetuses and infants and disturbances in neurodevelopment, thyroid, immune and reproductive systems. Additionally, these chemicals may exert genetic or epigenetic effects when metabolized.

On the other hand, in 1986, Barker and Osmond [5] suggested the relationship between poor nutrition in early life and later risk for ischemic heart disease. This observation, which had linked the importance of the intrauterine and early childhood nutritional environment and later disease risk, as well as dozens of additional epidemiological studies suggested a relationship between low birth weight and future risk of certain diseases such as cardiovascular disease, type 2 diabetes, obesity, schizophrenia and asthma. Today, these concepts have been expanded from birth weight to the entire fetal and infantile development, which led to the establishment of the Developmental Origin of Health and Disease (DOHaD) hypothesis [6, 7].

In light of these two groundbreaking concepts, there is a great concern that the consequences of intrauterine growth restriction or intrauterine insults caused by prenatal exposure to the environmental chemicals might linger throughout one's life.

Among the environmental chemicals, two of the most studied substances in environmental epidemiology are polychlorinated biphenyls (PCBs) and dioxins. Thus far, various cohort studies have been conducted to estimate the effects of these substances on fetal and infantile health. These studies inferred that prenatal exposure to $\mathrm{PCB} /$ dioxin could result in fetal growth restriction, cognitive and motor developmental retardation, disrupted sexual dimorphic behavior or reproductive health, and weakened immune systems [8-14].

Furthermore, as a result of recent growing concerns about the adverse health effects of perfluorinated compounds (PFCs), several epidemiological studies were conducted to evaluate the health effects of intrauterine PFCs exposure. Three studies reported correlations between prenatal PFOS/PFOA exposure and reduced birth weight [15-17]. Moreover, in a Danish study, the authors suggested that prenatal PFOA exposure could also increase the risk for obesity and the levels of insulin and leptin in females at 20 years of age [18], which was in line with the Developmental Origin of Health and Disease hypothesis. In addition, recent studies indicated that prenatal exposure to PFCs could also affect fetal and infantile thyroid function [19] as well as the immune system [20, 21].

These adverse health effects were considered to result from the endocrine disrupting activities of the environmental chemicals [3]. However, at this moment, the adverse health effects of prenatal exposures to the environmental chemicals are controversial; despite evidence from animal models, there is insufficient epidemiological evidence to substantiate this assertion $[9,11]$. In addition, this assertion is complicated by the fact that the effects of these chemicals in humans are still not fully understood. Many toxicological studies in animals suggest the dose-additive effects of chemicals acting on common endocrine pathways. However, it is challenging to estimate the effects of these compounds in humans that are constantly exposed to a wide variety of chemicals in their daily life [22].

Conversely, it is plausible that the adverse effects of the EDCs are attributed not only to their exposure dosage but also to the activities of the enzymes that metabolize these chemicals in the body and the binding affinity of these chemicals to their receptor, which may result in inhibition or facilitation of the expression of genes essential for human development. The function and expression levels of proteins are influenced by genetic factors such as single nucleotide polymorphisms (SNPs) and copy number variations (CNVs). Therefore, it is important to uncover genetic risk factors to environmental chemical exposure because there are currently few studies that take these factors into account. Thus, to clarify the effect of prenatal exposure to environmental chemicals on children's health, it is important to study the effects of exposure to EDCs via both genetic and environmental approaches as well as evaluating gene-environment interactions.

One of the examples of the gene-environment interactions is the etiology of hypospadias. Hypospadias is a common congenital anomaly caused by an incomplete fusion of the urethral folds. In our previous studies, we had clarified the etiology of hypospadias with genetic factors that were related to fetal endocrine activity such as the estrogen receptors (ESRI and 2) and 17 $\beta$-hydroxysteroid dehydrogenase type 3 (17ßHSD3) and maternal hormonal activity such as the cytochrome P450 1A1 (CYPIAl) in a retrospective case-control study [23-26]. Because development of the urethral and external genital system in the male fetus is androgen-dependent, abnormalities in the synthesis and metabolism of androgens resulting from EDCs exposure can result in abnormal genital development phenotypes.

However, there were some limitations in our previous studies. First, in the retrospective case-control study, there was not sufficient evidence to support a causal relationship between hypospadias and the environmental exposures because it was impossible to obtain relevant information about EDCs exposure levels prior to the study baseline. Second, there was both information and selection bias, i.e., recall bias, etc. Thus, we established the first large-scale birth cohort study in Japan in 2002 because of the need for investigating the effects of environmental exposures 
prospectively combined with genetic predispositions to evaluate the development and health of individuals from the prenatal period to adolescence (up to 13 years old) [26].

This study was primarily concerned with (1) examining the possible negative effects of perinatal environmental chemical exposures on birth outcomes, including congenital anomalies and growth retardation, (2) following the development of allergies, infectious diseases and neurodevelopmental disorders and performing a longitudinal observation of child development, (3) identifying a highrisk group classified by genetic susceptibility to environmental chemicals and (4) identifying the additive effects of various chemicals encountered in the daily environment.

The purpose of this review is to summarize the results of our recent studies and to address the necessary issues to be solved in the future.

\section{Methods}

Study areas and subjects

The Hokkaido study on Environment and Children's Health is an ongoing cohort study that began in 2002. The study consists of two prospective birth cohorts: the Sapporo (Toho hospital) cohort with one obstetric hospital in Sapporo City and the Hokkaido (large-scale) cohort with 37 hospitals and clinics in the Hokkaido prefecture. Hokkaido is the northern most prefecture and the second largest island of Japan; it has an area of $83,457 \mathrm{~km}^{2}$, equivalent to that of Austria. The population of Hokkaido is about 5.4 million, which is similar to that of Finland (Fig. 1).

The enrollment of the Sapporo cohort (Toho hospital) was conducted from July 2002 to October 2005. The subjects were women that were enrolled at 23-35 weeks of gestation and delivered at the Toho hospital. All of the subjects were residents of Sapporo City or surrounding areas.

From February 2003 through March 2012, the Hokkaido (large-scale) cohort had conducted the enrollment of women during early pregnancy $(<13$ weeks of gestational age) that visited one of the associated hospitals or clinics in the study area for prenatal health care in the maternity unit. This cohort consists of 20,940 pregnant women. In total, 37 hospitals and clinics in the Hokkaido prefecture participated in the study (the names of the hospitals are listed at the end of the paper). The study was conducted with the informed consent of all subjects in written form. The Institutional Ethical Board for Human Gene and Genome studies at Hokkaido University Center for Environmental and Health Sciences (CEHS) and Hokkaido University Graduate School of Medicine approved the study protocol.

Study design

The protocol for the study that is currently being conducted (subjects, outcomes and exposure measurement items) was partly described in the previous review [27]. In the current paper, the complete study design (up to 8 years), including the continued observation of subjects, has been described in Fig. 2 (the Sapporo cohort) and Fig. 3 (the Hokkaido large-scale cohort).

When examining subjects in the Sapporo cohort (Fig. 2), observations were focused on the association between child growth, neurodevelopment, allergy and infectious diseases, and low-level exposure to environmental chemicals during pregnancy and infancy. In this cohort, a self-administered questionnaire was completed at the time of enrolment to obtain baseline information including parental demographic characteristics, dietary
Fig. 1 The geographical distributions of the collaborating hospitals in Hokkaido, Japan. The large circled dot indicates Sapporo city (the prefectural capital of Hokkaido). The black dots indicate the geographical distributions of the collaborating hospitals and clinics outside of Sapporo City

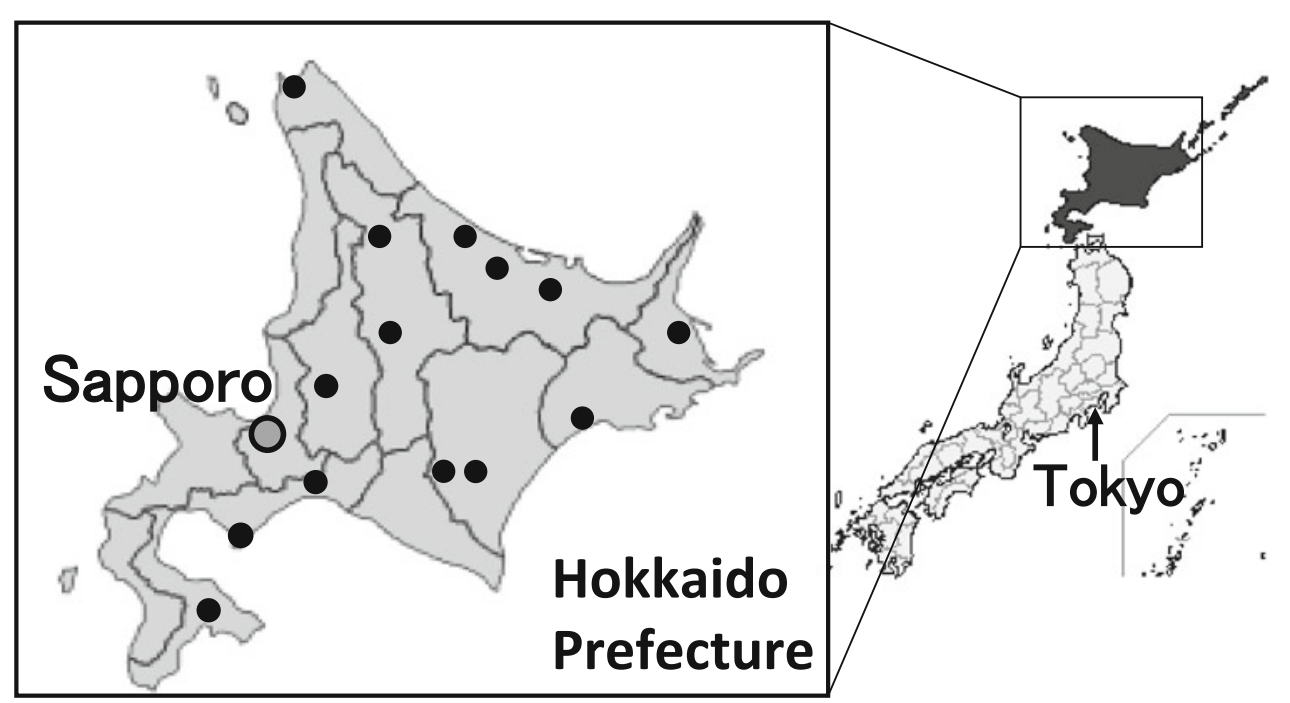




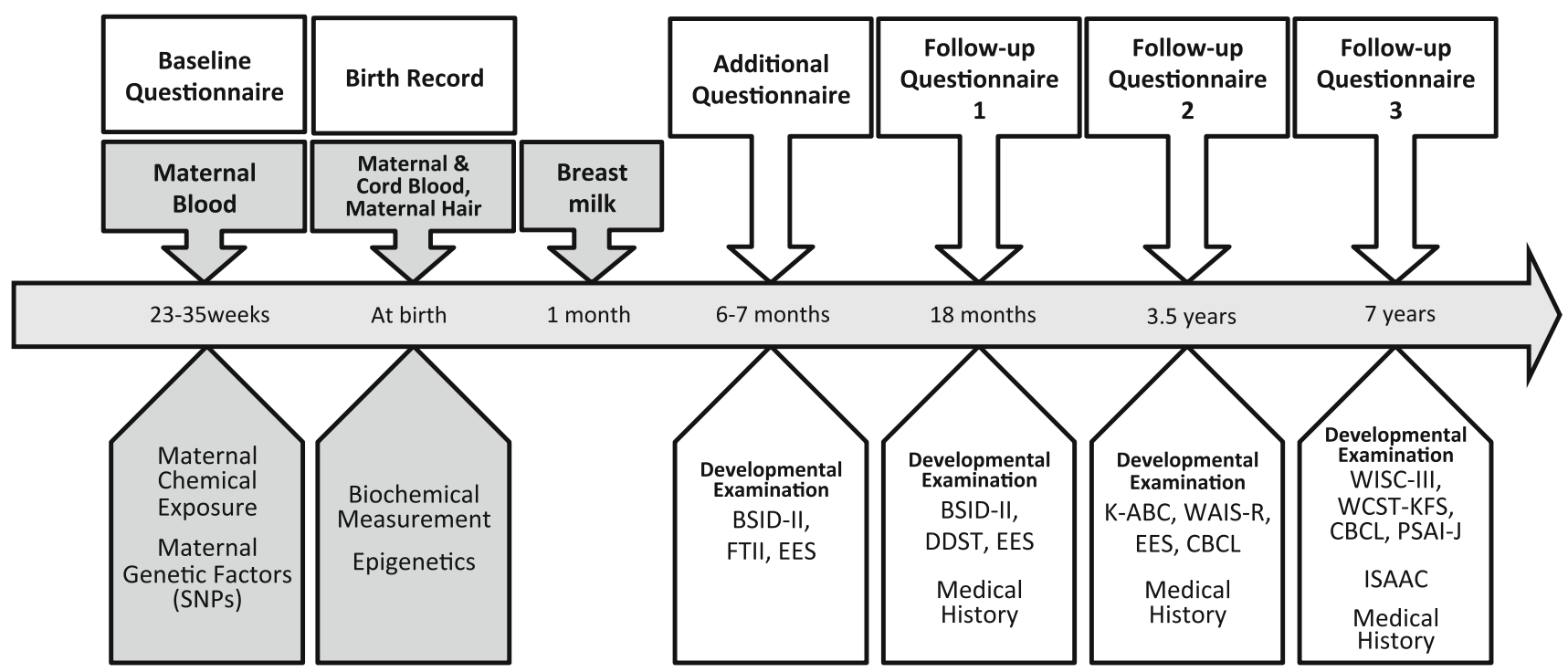

Fig. 2 Design of the Sapporo Toho hospital cohort study: obtaining information and specimens. SNPs single nucleotide polymorphisms, BSID-II The Bayley Scales of Infant Development-Second edition, FTII The Fagan Test of Infant Intelligence, EES the evaluation of environmental stimulation, DDST The Denver developmental screening tests, $K-A B C$ The Kaufman-Assessment Battery for Children,
$W A I S-R$ The Wechsler Adult Intelligence Scale-Revised, $C B C L$ Child Behavior Checklist, WISC-III The Wechsler Intelligence Scale for Children-Third edition, WCST-KFS Wisconsin Card Sorting TestKeio-F-S version, PSAI-J Pre-School Activities Inventory-Japanese version, ISAAC International Study of Asthma and Allergies in Childhood

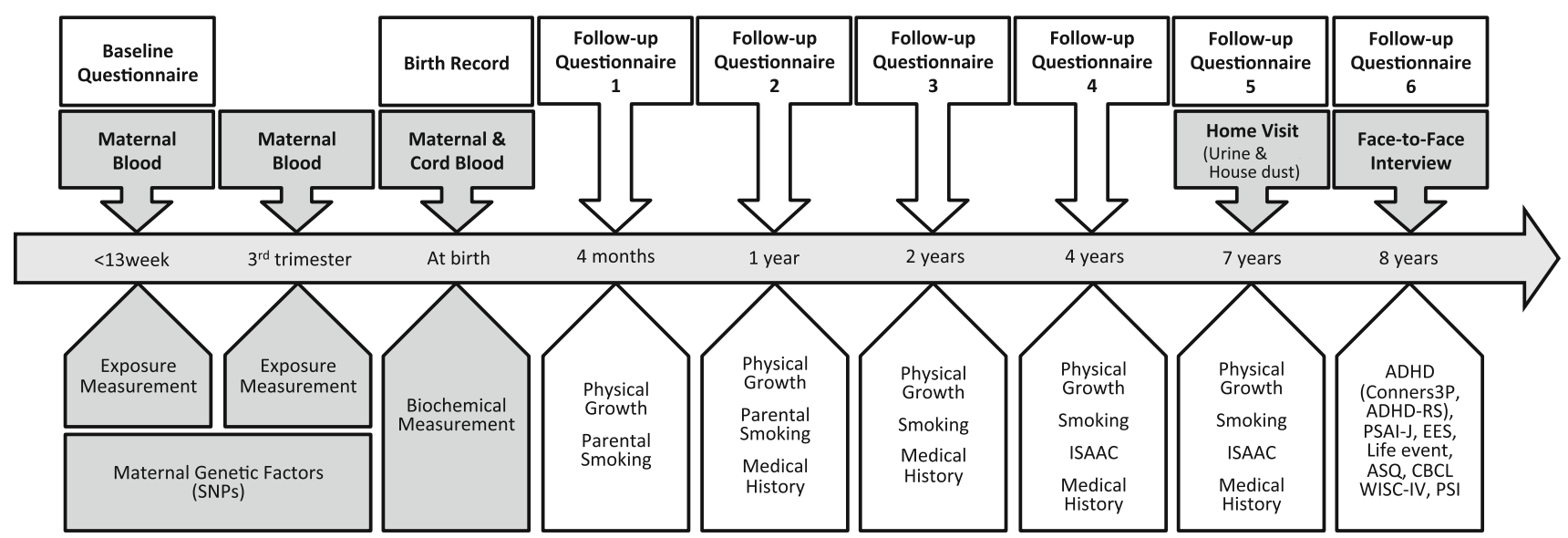

Fig. 3 Design of the Hokkaido large-scale cohort study: obtaining information and specimens. SNPs single nucleotide polymorphisms, ISAAC International Study of Asthma and Allergies in Childhood, $A D H D$ attention deficit hyperactivity disorder, Conners $3 P$ The Conners Third edition Parent, $A D H D-R S$ attention deficit hyperactivity disorder-rating scale, PSAI-J Pre-School Activities Inventory-

habits including the amount and species of fish consumed, exposure to chemical compounds in their daily life, smoking history, alcohol consumption, caffeine intake and household income. Information on pregnancy complications, gestational age at birth, infant gender and birth size was obtained from maternal and infant medical records.

Follow-up questionnaires were also used at 18 months, 3.5 and 7 years of age to obtain relevant information including allergies, dietary habits and the smoking history of mother and her partner. Additionally, in the follow-up
Japanese version, EES the evaluation of environmental stimulation, Life Event life event questionnaire for parents, $A S Q$ autism screening questionnaire, $C B C L$ child behavior checklist, WISC-IV The Wechsler Intelligence Scale for Children-Fourth edition, PSI Parenting Stress Index

questionnaires, we also obtained information pertaining to the medical history of the children such as atopic dermatitis, asthma, allergies, otitis media, pneumonia or bronchitis and chickenpox. At 18 months and 3.5 years of age, infants were defined as having allergies or an infection if there was a diagnosis from a doctor, the infant was hospitalized or the infant received medical treatment. At 7 years of age, the International Study of Asthma and Allergies in Childhood (ISAAC) criteria was used to determine if the children had allergies or an infection [28]. 
In addition, we followed the neurodevelopment of the children using several behavioral examinations at 6-7, 18 months, 3.5 and 7 years of age to assess the effect of low-level intrauterine exposure to toxic chemicals on childhood neurodevelopment. More detailed protocols and information regarding neurodevelopmental examinations are described in the "Outcome Measurement" section below.

The Hokkaido cohort (Fig. 3) was established to assess the prevalence of congenital anomalies including cleft lip and palate, congenital heart defects, hypospadias and cryptorchidism. In addition, this cohort was used to explore the possible causes of these malformations, as well as the prevalence of childhood allergies and neurodevelopmental disorders including Attention Deficit Hyperactivity Disorder (ADHD). In this cohort, a baseline questionnaire survey was conducted at the time of enrollment during the first trimester to obtain parental information such as demographic characteristics, medical and obstetric history, dietary supplement intake during pregnancy, smoking history, alcohol and coffee consumption and chemical exposures at work. Perinatal data such as birth weight, infant gender, mode of delivery, multiple conception and the diagnosis of congenital anomalies were obtained from birth records completed by an obstetrician. We classified 55 congenital anomalies as "representative congenital anomalies" according to the classification by Konishi [29] with some additional anomalies by our study group such as congenital heart diseases, hypospadias, cryptorchidism etc., to study the effect of mutagens and teratogens among EDCs. The first follow-up questionnaire was used on infants at 4 months of age to obtain relevant data including birth size, gestational age at birth and parental smoking history during the second and third trimester. The successive follow-up questionnaires were administered at 1,2, 4, 7 and 8 years of age to obtain relevant information such as child height and weight measurements obtained at regular health checkups, vaccination history, dietary habits and parental smoking history. In these follow-up questionnaires, we also asked about the medical history of the children. Specifically, we determined if the children developed atopic dermatitis, asthma, allergies, otitis media, pneumonia or bronchitis, chickenpox, heart disease, hypospadias or cryptorchidism, thyroid gland malfunction, epilepsy or developmental disorders. At 1, 2, 4 and 7 years old, allergy and infection information was obtained by using the International Study of Asthma and Allergies in Childhood (ISAAC) [28] and the American Thoracic Society-Division of Lung Disease (ATS-DLD) [30]. At 1 and 2 years of age, infants were defined as having allergies or an infection if there was a diagnosis by a doctor, the infant was hospitalized or the infant received medical treatment. At 8 years old, several examinations for neurodevelopmental disorders were conducted to investigate the effects of perinatal and postnatal chemical exposure on children's health, specifically neurodevelopment.

Specimen collection and biochemical measurements

In the Sapporo cohort, maternal blood samples were collected during late pregnancy, usually after the 30th week of gestation. Cord blood and placenta were taken immediately after birth. Maternal hair samples were also collected within 5 days following delivery, and breast milk from nursing mothers was collected within 4 weeks following birth. In the Hokkaido cohort, maternal blood was collected 3 times: between 6 and 14 weeks of gestational age to represent the organogenetic period, during the third trimester and at delivery. Cord blood was taken immediately after birth in the same manner as done in the Sapporo cohort study.

The items that were measured biochemically from the specimens are described in Table 1. In the Sapporo cohort, the levels of cord serum immunoglobulin E (IgE) and immunoglobulin A (IgA) were also determined [31]. Thyroid stimulating hormone (TSH) and free thyroxine (FT4) levels of the mother and newborn were measured as part of a mass-screening program conducted in Sapporo

Table 1 Items measured in the Hokkaido study on environment and children's health

\begin{tabular}{ll}
\hline Specimen & Measurement \\
\hline Exposure measurement \\
Maternal blood & PCB and dioxin; PCDD and PCDF (congeners) \\
& OH-PCB (congener level) \\
& PFCs (PFOS, PFOA and other PFAAs) \\
& MEHP (phthalate metabolite) \\
& Chlorinated pesticides \\
& Cotinine \\
Maternal hair & Me-Hg \\
Cord blood & BPA \\
Child urine & Cotinine \\
& Phthalate and phosphate esters (7-year-old) \\
House dust & Phthalate and phosphate esters (7-year-old) \\
Biochemical measurements \\
Maternal blood & TSH, FT4, Folic acid, 11 Fatty acids \\
Cord blood & IgE, TSH, FT4, 9 Steroid hormones \\
\hline
\end{tabular}

$\overline{P C B}$ polychlorinated biphenyls, $P C D F$ polychlorinated dibenzofurans, $P C D D$ polychlorinated dibenzodioxins, $O H-P C B$ hydroxylated polychlorinated biphenyl, $P F C s$ perfluorinated compounds, $P F O S$ perfluorooctane sulfonate, $P F O A$ perfluorooctanoic acid, $P F A A s$ perfluoroalkyl acids, $M E H P$ mono-2-ethylhexyl phthalate, $\mathrm{Me}-\mathrm{Hg}$ methylmercury, BPA bisphenol A, TSH thyroid stimulating hormone, FT4 free thyroxine 
City. The levels of 9 key sex hormones in the cord blood (e.g., Estradiol, Testosterone, Progesterone, etc.) and the levels of 11 fatty acids in the maternal plasma were also measured by liquid chromatography-tandem mass spectrometry (LC-MS/MS) and gas chromatography-mass spectrometry (GC-MS), respectively. In the Hokkaido cohort, maternal serum was used to measure folic acid levels [32].

Exposure measurements

PCBs, OH-PCBs and Dioxins

In the Sapporo cohort, the levels of 29 congeners of dioxins and dioxin-like polychlorinated biphenyls (DL-PCBs) [7 polychlorinated dibenzodioxins (PCDDs), 10 polychlorinated dibenzofurans (PCDFs), 4 Non-ortho PCBs and 8 Mono-ortho PCBs], 58 congeners of the other PCBs and 5 congeners of hydroxylated polychlorinated biphenyls $(\mathrm{OH}-$ PCBs) in maternal blood and breast milk were measured using a high-resolution gas chromatography/high-resolution mass spectrometer (HRGC/HRMS) at the Fukuoka Institute of Health and Environmental Sciences [33-37]. The Toxicity Equivalency Quantity (TEQ) levels were calculated by multiplying the levels of individual congeners by its toxic equivalency factor (TEF) values of WHO 2005 [38].

\section{PFCs}

In the Sapporo cohort, PFOS and PFOA levels in maternal blood, cord blood and breast milk were analyzed by LCMS/MS at Hoshi University [39, 40]. For the Hokkaido cohort study, among PFCs, 11 perfluoroalkyl acids (PFAAs) [perfluorohexanoic acid (PFHxA), perfluoroheptanoic acid (PFHpA), perfluorooctanoic acid (PFOA), perfluorononanoic acid (PFNA), perfluorodecanoic acid (PFDA), perfluoroundecanoic acid (PFUnDA), perfluorododecanoic acid (PFDoDA), perfluorotridecanoic acid (PFTrDA), perfluorotetradecanoic acid (PFTeDA), perfluorohexane acid (PFHxS) and perfluorooctane sulfonate (PFOS)] were measured in maternal plasma using simultaneous analysis with ultraperformance liquid chromatography in combination with triple quadrupole mass spectrometry (UPLC-MS/MS) at the Research Faculty of Agriculture, Hokkaido University [41].

\section{Organochlorine pesticides}

In the Sapporo cohort, the levels of persistent organochlorine pesticides in maternal blood were analyzed by a gas chromatography/negative-ion chemical-ionization mass spectrometry (GC/NCIMS) and a gas chromatography/high-resolution mass spectrometry (GC/HRMS) at IDEA Consultants, Inc. [42].

\section{Metals}

In the Sapporo cohort, total mercury levels in maternal hair samples were measured by an oxygen combustion-gold amalgamation method using an atomic absorption detector at the National Institute for Minamata Disease [43, 44].

\section{Phthalate esters and organophosphate flame retardants}

In the Sapporo cohort, to determine maternal phthalate exposure levels, MEHP (a metabolite of DEHP) levels in maternal blood were analyzed by GC-MS at Nagoya University [45]. In the Hokkaido cohort, 7 phthalates and 11 organophosphate flame-retardants were measured from dust samples using GC-MS (SIM) analysis. House dust mites were also measured using the ELISA method. The method to analyze 7 phthalate metabolites in urine samples by GC-MS was established, and urine samples from the children were measured to examine the correlation between these metabolites and asthma and allergies [46, 47]. Home visits were also conducted for the children that lived in Sapporo City. During the home visit, house dust and urine samples from the child were collected. In addition, trained researchers evaluated the home interior and dampness.

\section{Bisphenol A}

In the Sapporo cohort, Bisphenol A concentrations in maternal and cord blood were analyzed by isotope dilutionliquid chromatography-tandem mass spectrometry (IDLC-MS/MS) at IDEA Consultants, Inc. [48].

\section{Cotinine}

In the Sapporo and the Hokkaido cohorts, cotinine concentrations in maternal serum were measured using an enzyme-linked immunosorbent assay (ELISA) kit to evaluate smoking exposure levels [49].

Outcome measurements

\section{The Sapporo cohort}

In the Sapporo cohort, with the purpose of assessing the neurodevelopment of the children, several behavioral examinations were conducted during each study period. The Bayley Scales of Infant Development second edition (BSID-II) was used at 6-7 and 18 months of age. The 
Fagan Test of Infant Intelligence (FTII) was performed to measure visual recognition memory and cognitive ability in infants aged 6-7 months. To examine developmental progress, the Japanese version of the Denver Developmental Screening Tests (DDST) was used at 18 months of age. At 3.5 years of age, child and maternal intelligence was measured using the Japanese version of the Kaufman Assessment Battery for Children (K-ABC) and the Wechsler Adult Intelligence Scale-Revised (WAIS-R), respectively. At 7 years of age, the Wechsler Intelligence Scale for Children third edition (WISC-III) and the Wisconsin Card Sorting Test (WCST-KFS version) were used to assess the intellectual development and executive function of the children [50, 51]. The Evaluation of Environmental Stimulation (EES) was used to investigate the environmental conditions of children at 6,18 months and 3.5 years of age. The Japanese version of the Child Behavior Checklist (CBCL) was used to collect information on child behavior at age 3.5 and 7 years of age. The check list of play behavior, Pre-School Activity InventoryJapanese version (PSAI-J), which was translated from the original version of PSAI, was used to assess the play behavior of the children at 7 years of age [52]. In addition, we also obtained the children's medical history from the follow-up questionnaires performed at each study period. The children's medical history contained information pertaining to the development of atopic dermatitis, asthma, allergies, otitis media, pneumonia or bronchitis and chickenpox.

\section{The Hokkaido cohort}

In the Hokkaido cohort, the development of allergies at 1, 2, 4 and 7 years of age and neurodevelopmental disorders at 8 years of age were examined in detail. For allergy assessment, follow-up questionnaires were distributed to children aged 1, 2, 4 and 7 years old, which included questions pertaining to asthma and allergies from the ISAAC and ATS-DLD questionnaires [28, 30]. We also obtained the medical history of the children from the follow-up questionnaires during each study period. The medical histories contained information pertaining to the development of atopic dermatitis, asthma, allergies, otitis media, pneumonia or bronchitis, chickenpox, heart disease, hypospadias or cryptorchidism, thyroid gland malfunction, epilepsy and developmental disorders. In addition to the questionnaire survey, mothers were asked to collect house dust and a sample of the child's urine when the child reached 7 years old.

At 8 years of age, a specific follow-up questionnaire was used to assess the development of neurodevelopmental disorders, specifically ADHD. The questionnaire contained questions pertaining to health status including the treatment the subject received for ADHD, the hours of rising and bedtime as a daily rhythm, and the number of hours the subject enjoys audio-visual tools. To assess ADHD, the Conners third Edition-Parent Japanese version (Conners3P) and the ADHD Rating Scale-IV (ADHD-RS-IV) were used. We also used the Pre-School Activities Inventory Japanese version (PSAI-J) to assess the play behavior of the children. The Evaluation of Environmental Stimulation (EES) was used as a questionnaire to assess the subject's home environment [53]. We also assessed any stressful life events of the children by using the Life Event Questionnaire for Parents (Life Event) [54].

After receiving responses from the 8-year questionnaire, additional questionnaires were distributed to collect more information about the family. The additional questionnaire assessed the working status and health of the parents, the mental condition of the mother, and the use or lack of use of supportive education. To assess a child-rearing environment, we asked about the parent's social networks and supports during child rearing. To assess developmental disorders such as Autism and Asperger syndrome, we used the Japanese version of the Autism screening Questionnaire (ASQ). Additional assessments of the children we obtained using the Japanese version of the Child Behavior Checklist (CBCL) and The Wechsler Intelligence Scale for Children fourth edition (WISC-IV). We also used the Parenting Stress Index (PSI) in Japanese.

\section{Genetic analyses}

Genes that were already analyzed using the SNP assay are described in Table 2. Genetic polymorphisms were determined by means of the Taq Man (Applied Biosystems, Inc., Foster City, CA, USA) polymerase chain reaction (PCR) method using minor groove binder (MGB) probes. The polymorphisms analyzed thus far are rs4646903 ( $\mathrm{T}>\mathrm{C}$, MspI) and rs1048963 (A $>\mathrm{G}$, Ile462Val) of CYP1A1 (cytochrome P450, family 1, subfamily A polypeptide 1), rs762551 (A > C) of CYP1A2 (CYP1 subfamily A polypeptide 2), rs1056836 (C> G, Leu432Val) of CYP1B1 (CYP1 subfamily B polypeptide 1), rs2066853 (G > A, Arg554Lys) of $A H R$ (aryl hydrocarbon receptor), rs2292596 (C > G, Pro185Ala) of AHRR (AHR repressor), rs1800566 (C609T) of NQO1 (NAD(P)H: quinone oxidoreductase 1), rs3813864 (-1294G/C) of CYP2E1 (CYP2 subfamily E polypeptide 1), rs1801133 (C677T) and rs1801131 (A1298C) of MTHFR (methylenetetrahydrofolate reductase). In addition, copy number variations (CNVs) in GSTM1 (glutathione S-transferase mu-1) and GSTT1 (glutathione S-transferase theta-1) were also evaluated [55-58]. 
Table 2 Genetic factors and its environmental interaction being studied in the Hokkaido study (up to 2013)

\begin{tabular}{|c|c|c|c|c|}
\hline Maternal genetic factors & Environmental exposure & Outcomes & Results & Ref. \\
\hline AHR, AHRR, CYP1A1, CYP1A2, CYP1B1 & Dioxin and dioxin-like PCBs & (Concentration) & Decreased & [55] \\
\hline AHR, CYP1A1, GSTM1, GSTT1 & Active tobacco smoking (PAHs) & Birth size & Reduction & [56] \\
\hline NQO1, CYP2E1, MGMT & Active tobacco smoking & Birth size & Reduction & [57] \\
\hline 5,10-MTHFR (C677T, A1298C) & Tobacco smoking and Folic acid & Birth weight & Reduction & [58] \\
\hline
\end{tabular}

Genes described in bold font in the table represent the genetic polymorphisms that are significantly associated with the outcome

$A H R$ aryl hydrocarbon receptor, $A H R R$ AHR repressor, $C Y P 1$ cytochrome P450, family 1, CYP1A1 CYP1 subfamily A polypeptide 1, CYP1A2 CYP1 subfamily A polypeptide 2, CYP1B1 CYP1 subfamily B polypeptide 1, GSTM1 glutathione-S-transferase mu-1, GSTT1 glutathione-S-transferase theta1, NQO1 NAD(P)H: quinone oxidoreductase 1, CYP2E1 CYP2 subfamily E polypeptide 1, MTHFR methylenetetrahydrofolate reductase

\section{Results}

The characteristics of the participants of the Hokkaido study

A total of 514 mothers were registered in the Sapporo cohort, and another 20,940 mothers were registered in the Hokkaido cohort as of the end of April 2012. The profile of the Sapporo cohort and the partial profile $(n=2,777)$ of the Hokkaido cohort had been described previously [27]. We also estimated the prevalence of congenital anomalies in the Hokkaido prefecture. Among the 19,680 mothers included in the Hokkaido cohort between 2003 and 2012, there were 378 subjects with congenital anomalies. The most frequent congenital anomaly was congenital heart defects (35.6 per 10,000 persons), followed by cryptorchidism (15.2), down syndrome (12.2), polydactyly (9.7), hypospadias (9.1) and hydronephrosis (7.6). The total prevalence of congenital anomalies was similar to nationwide data reported by the Japan Association of Obstetricians and Gynecologists (JAOG). However, the number of serious cases was less than that of the JAOG since the members of the JAOG are medical universities and tertiary hospitals and they tend to treat pregnant women with severe complications including fetal congenital anomalies, whereas those of our cohort study are general hospitals and clinics.

The effects of PCDD/PCDF and dioxin-like PCB exposure

\section{Birth weight}

In the Sapporo cohort, we observed significant negative correlations between the birth weight of all infants and total PCDF levels, total PCDD TEQ, total PCDF TEQ, total PCDD/PCDF TEQ and total TEQ levels in maternal blood during pregnancy after adjustment for potential covariates. Among male infants, significant adverse associations
Table 3 Gender differences in the effect of PCB/dioxins exposure on birth weight in a multiple linear regression model

\begin{tabular}{|c|c|c|c|c|}
\hline \multirow[t]{2}{*}{$\log _{10}$ scale } & \multicolumn{2}{|l|}{ Male } & \multicolumn{2}{|l|}{ Female } \\
\hline & $\beta^{\mathrm{a}}$ & $p$ value & $\beta^{\mathrm{a}}$ & $p$ valu \\
\hline \multicolumn{5}{|l|}{ Total (pg/g lipid) } \\
\hline Total PCDDs & -125.7 & 0.371 & -19.3 & 0.890 \\
\hline Total PCDFs & -237.6 & 0.191 & -304.9 & 0.058 \\
\hline Total PCDDs/PCDFs & -136.6 & 0.340 & -28.7 & 0.839 \\
\hline Total non-ortho PCBs & -90.7 & 0.491 & -122.4 & 0.286 \\
\hline Total mono-ortho PCBs & -138.6 & 0.244 & -104.3 & 0.315 \\
\hline Total DL-PCBs & -138.7 & 0.245 & -105.3 & 0.311 \\
\hline Total dioxin & -148.5 & 0.229 & -106.8 & 0.319 \\
\hline \multicolumn{5}{|c|}{ TEQ (WHO 2005) ${ }^{\dagger}$ (TEQ pg/g lipid) } \\
\hline Total PCDDs TEQ & -331.4 & $0.019 *$ & -126.3 & 0.336 \\
\hline Total PCDFs TEQ & -269.8 & 0.070 & -241.7 & 0.058 \\
\hline Total PCDDs/PCDFs TEQ & -338.7 & $0.022 *$ & -173.9 & 0.195 \\
\hline Total non-ortho PCBs TEQ & -107.3 & 0.288 & -114.8 & 0.196 \\
\hline $\begin{array}{l}\text { Total mono-ortho PCBs } \\
\text { TEQ }\end{array}$ & -138.6 & 0.244 & -104.3 & 0.315 \\
\hline Total DL-PCBs TEQ & -112.1 & 0.278 & -117.5 & 0.195 \\
\hline Total dioxin TEQ & -289.5 & $0.037 *$ & -144.2 & 0.243 \\
\hline
\end{tabular}

This table was reconstructed by using data from a previously published study by Konishi et al. [59]. Among male infants, a significant negative association between birth weight and total PCDDs TEQ levels, total PCDDs/PCDFs TEQ levels and total TEQ levels was found. However, among the female infants, these significant associations were not found

$* p<0.05$

${ }^{\dagger}$ The Toxicity Equivalency Quantity (TEQ) levels were calculated by multiplying the levels of individual congeners by its toxic equivalency factor (TEF) values of WHO 2005 [38]

${ }^{a}$ Beta coefficients represent the change in birth weight $(\mathrm{g})$ for a 10 -fold increase in the levels of PCDDs/PCDFs and DL-PCBs

between birth weight and total PCDD TEQ levels, total PCDD/PCDF TEQ levels and total TEQ levels were found. Moreover, we found significant negative association between birth weight and the levels of 2,3,4,7,8-PeCDF (-24.5 g, $95 \%$ CI -387.4 to -61.5) [59] (Table 3). 


\section{Neurodevelopment}

In the Sapporo cohort, after adjusting for potential confounding variables, total PCDD, total PCDDs/PCDF and $1,2,3,4,6,7,8$-HpCDD levels in maternal blood during pregnancy were significantly negatively associated with the mental developmental index (MDI) of BSID-II at 6 months of age. Total 1,2,3,7,8,9-HxCDD, 1,2,3,4,6,7,8-HpCDD, $2,3,7,8-\mathrm{TCDF}, 1,2,3,7,8-\mathrm{PeCDF}$ and $1,2,3,6,7,8-\mathrm{HxCDF}$ were significantly negatively associated with the psychomotor developmental index (PDI) of BSID-II at 6 months of age. Our results suggest that a low-level of exposure to several congeners of PCDDs or PCDFs during pregnancy can affect the neurodevelopment of 6-month-old infants [60]. In addition, when we stratified the data by infant sex, the effects of intrauterine exposure to select PCDD, PCDF and PCB congeners on the PDI score in male infants were more significant (Table 4).

\section{Allergy and infectious diseases}

In the Sapporo cohort, our results show that dioxins concentrations in maternal blood during pregnancy are only negatively correlated with cord serum IgE levels in male infants [61] (Table 5). Relatively higher levels of PCDFs were associated with a significantly increased risk of otitis media at 18 months of age, among all infants (odds ratio $=2.5,95 \%$ confidence interval $=1.1-5.9$ ). Relatively higher levels of 2,3,4,7,8-PeCDF were also associated with a significantly increased risk of otitis media (odds ratio $=5.3,95 \%$ confidence interval $=1.5-19$ ) among male infants (Table 6). However, we observed a weak association between dioxin-like compound levels and allergy symptoms during infancy. At environmental levels, prenatal exposure to dioxin-like compounds may alter immune function and increase the risk of infections in infancy, especially among males. The compound 2,3,4,7,8PeCDF may be responsible for this [62].

The effects of PFCs exposure

\section{Temporal trends of PFC levels in maternal plasma}

In the Sapporo cohort, the concentrations of PFOS and PFOA ranged from 1.3 to $16.2 \mathrm{ng} / \mathrm{ml}$ for PFOS and from below the detection limit to $5.3 \mathrm{ng} / \mathrm{ml}$ for PFOA (both detection limits were $0.5 \mathrm{ng} / \mathrm{ml}$ ) in the blood of pregnant women recruited between 2002 and 2005 [63].

In the Hokkaido cohort, between February 2003 and December 2009, 300 women were randomly selected every year, and the concentrations of $11 \mathrm{PFCs}$ were measured in 2,095 maternal plasma samples. A temporal trend in PFC levels from 2003 to 2011 was also examined. The PFOS and PFOA concentrations in the Hokkaido cohort were lower than those of pregnant women in the Sapporo cohort. Additionally, PFUnDA, PFDoDA and PFTrDA levels were higher in the Hokkaido cohort than individuals of foreign countries. Although the values were lower than the values obtained from individuals in other areas of Japan, there was no significant temporal trend [64].

\section{Birth weight}

We examined a correlation between maternal serum PFOS and PFOA concentrations and infant birth weight in the Sapporo cohort. A $\log _{10}$-unit increase in PFOS levels correlated with a decrease in birth weight of $148.8 \mathrm{~g} \mathrm{(95 \%}$ CI 297.0-0.5) after adjusting for confounders; however, no correlation was observed between PFOA levels and birth weight. Our results indicate that in utero exposure to relatively low levels of PFOS is negatively correlated with birth weight [63].

In the Hokkaido cohort, the effects of 11 PFCs including PFHxA, PFHpA, PFHxS, PFOS, PFOA, PFNA, PFDA, PFUnDA, PFDoDA, PFTrDA and PFTeDA in maternal blood obtained during pregnancy were evaluated. After adjusting for possible confounding factors, PFNA levels negatively correlated with birth weight (per ln-unit: partial regression coefficient $\beta=-41.7 \mathrm{~g}, 95 \% \mathrm{CI},-77.9$ to $-5.6 \mathrm{~g}$ ). After gender stratification, PFNA levels negatively correlated with male birth weight (per ln-unit: $\beta=-59.3 \mathrm{~g}$, $95 \% \mathrm{CI},-110.2$ to $-8.3 \mathrm{~g}$ ). Additionally, PFUnDA and PFTrDA levels negatively correlated with female birth weight (per ln-unit: $\beta=-42.0 \mathrm{~g}, 95 \% \mathrm{CI},-84.6$ to $0.6 \mathrm{~g}$ and $\beta=-44.9 \mathrm{~g}, 95 \% \mathrm{CI},-90.1$ to $0.3 \mathrm{~g}$, respectively).

\section{Allergy and infectious diseases}

In the Sapporo cohort, we investigated the relationship between prenatal exposure to PFOS and PFOA and the development of infant allergies and infectious diseases during the first 18 months of life. Additionally, the effects of PFOS and PFOA on cord blood IgE levels were also evaluated. We found a curvilinear relationship between maternal PFOA levels and cord blood IgE levels. Cord blood IgE levels decreased significantly with high maternal PFOA concentrations among female infants. When $\log _{10^{-}}$ transformed maternal PFOA levels changed from 0.3 to $0.7 \mathrm{ng} / \mathrm{mL}, \log _{10}$-transformed cord blood IgE levels greatly decreased by $-0.863 \mathrm{IU} / \mathrm{mL}$. However, there were no significant associations among maternal PFOS and PFOA levels and food allergies, eczema, wheezing or otitis media in the 18-month-old infants after adjustment for potential confounding variables [31].

In the Hokkaido cohort, we investigated the relationship between prenatal exposure to 11 PFCs and infant 
Table 4 Gender differences in the effect of PCB/dioxins exposure on BSID-II Mental (MDI) and Psychomotor (PDI) development scores at 6 months of age in multiple linear regression models

\begin{tabular}{|c|c|c|c|c|c|c|c|c|c|c|c|c|}
\hline \multirow[t]{3}{*}{$\left(\log _{10}\right.$ transformed) } & \multicolumn{6}{|c|}{6 months MDI } & \multicolumn{6}{|c|}{6 months PDI } \\
\hline & \multicolumn{3}{|c|}{ Male $(n=99)$} & \multicolumn{3}{|c|}{ Female $(n=91)$} & \multicolumn{3}{|c|}{ Male $(n=99)$} & \multicolumn{3}{|c|}{ Female $(n=91)$} \\
\hline & $\beta^{\mathrm{a}}$ & $t$ & $p$ value & $\beta^{\mathrm{a}}$ & $t$ & $p$ value & $\beta^{\mathrm{a}}$ & $t$ & $p$ value & $\beta^{\mathrm{a}}$ & $t$ & $p$ value \\
\hline \multicolumn{13}{|l|}{ PCDD } \\
\hline 2,3,7,8-TCDD & -0.15 & -1.54 & 0.13 & -0.05 & -0.48 & 0.63 & -0.19 & -2.01 & $0.048 *$ & -0.06 & -0.56 & 0.58 \\
\hline 1,2,3,7,8-PeCDD & -0.07 & -0.70 & 0.48 & 0.22 & 2.14 & $0.04 *$ & -0.10 & -0.98 & 0.33 & -0.04 & -0.33 & 0.75 \\
\hline $1,2,3,4,6,7,8-\mathrm{HpCDD}$ & -0.25 & -2.52 & $0.01 *$ & -0.14 & -1.34 & 0.18 & -0.24 & -2.56 & $0.01 *$ & -0.19 & -1.78 & 0.08 \\
\hline OCDD & -0.09 & -0.92 & 0.36 & -0.18 & -1.74 & 0.09 & -0.22 & -2.33 & $0.02 *$ & -0.21 & -1.97 & 0.05 \\
\hline \multicolumn{13}{|l|}{ PCDF } \\
\hline $2,3,7,8-\mathrm{TCDF}$ & -0.08 & -0.84 & 0.41 & -0.11 & -1.05 & 0.30 & -0.21 & -2.21 & $\mathbf{0 . 0 3} *$ & -0.13 & -1.21 & 0.23 \\
\hline $1,2,3,7,8-\mathrm{PeCDF}$ & -0.02 & -0.22 & 0.83 & -0.06 & -0.54 & 0.59 & -0.22 & -2.38 & $0.02 *$ & -0.17 & -1.59 & 0.12 \\
\hline $1,2,3,4,7,8-\mathrm{HxCDF}$ & -0.07 & -0.73 & 0.47 & -0.10 & -0.93 & 0.36 & -0.17 & -1.69 & 0.09 & -0.25 & -2.36 & $0.02 *$ \\
\hline \multicolumn{13}{|l|}{ Non-ortho PCB } \\
\hline $33^{\prime} 44^{\prime} 5$-PenCB (\#126) & -0.03 & -0.33 & 0.74 & -0.01 & -0.10 & 0.93 & -0.15 & -1.62 & 0.11 & -0.24 & -2.25 & $\mathbf{0 . 0 3} *$ \\
\hline \multicolumn{13}{|l|}{ Mono-ortho PCB } \\
\hline 2344'5-PeCB (\#114) & -0.07 & -0.71 & 0.48 & 0.08 & 0.79 & 0.43 & -0.19 & -2.00 & $0.049 *$ & -0.16 & -1.49 & 0.14 \\
\hline $2^{\prime} 344^{\prime} 5-\mathrm{PeCB}(\# 123)$ & 0.02 & 0.23 & 0.82 & 0.01 & 0.05 & 0.96 & -0.13 & -1.39 & 0.17 & -0.25 & -2.37 & $0.02 *$ \\
\hline $233^{\prime} 44^{\prime} 5^{\prime}-\mathrm{HxCB}(\# 157)$ & -0.08 & -0.85 & 0.40 & 0.10 & 0.90 & 0.37 & -0.21 & -2.19 & $\mathbf{0 . 0 3} *$ & -0.11 & -1.09 & 0.28 \\
\hline $23^{\prime} 44^{\prime} 55^{\prime}-\mathrm{HxCB}(\# 167)$ & -0.05 & -0.49 & 0.63 & 0.04 & 0.41 & 0.69 & -0.22 & -2.35 & $0.02 *$ & -0.15 & -1.38 & 0.17 \\
\hline \multicolumn{13}{|l|}{ Di-ortho PCB } \\
\hline $22^{\prime} 33^{\prime} 44^{\prime} 5-\mathrm{HpCB}(\# 170)$ & -0.13 & -1.25 & 0.22 & 0.10 & 0.88 & 0.38 & -0.25 & -2.47 & $0.02 *$ & -0.04 & -0.37 & 0.71 \\
\hline $22^{\prime} 344^{\prime} 55^{\prime}-\mathrm{HpCB}(\# 180)$ & -0.13 & -1.23 & 0.22 & 0.10 & 0.88 & 0.38 & -0.24 & -2.42 & $0.02 *$ & 0.00 & 0.01 & 1.00 \\
\hline \multicolumn{13}{|l|}{ Total } \\
\hline Total PCDD & -0.10 & -1.00 & 0.32 & -0.17 & -1.63 & 0.11 & -0.22 & -2.31 & $0.02 *$ & -0.21 & -1.97 & 0.05 \\
\hline Total PCDF & -0.06 & -0.61 & 0.55 & 0.02 & 0.15 & 0.88 & -0.18 & -1.81 & 0.07 & -0.20 & -1.83 & 0.07 \\
\hline Total PCDD/PCDF & -0.10 & -1.00 & 0.32 & -0.17 & -1.58 & 0.12 & -0.22 & -2.33 & $0.02 *$ & -0.21 & -1.98 & 0.05 \\
\hline Total non-ortho PCBs & -0.01 & -0.12 & 0.91 & 0.03 & 0.25 & 0.81 & -0.16 & -1.72 & 0.09 & -0.19 & -1.73 & 0.09 \\
\hline Total mono-ortho PCBs & -0.05 & -0.55 & 0.58 & 0.05 & 0.46 & 0.64 & -0.19 & -1.97 & 0.05 & -0.17 & -1.60 & 0.11 \\
\hline Total DL-PCB & -0.05 & -0.55 & 0.59 & 0.05 & 0.46 & 0.65 & -0.19 & -1.97 & 0.05 & -0.17 & -1.60 & 0.11 \\
\hline Total dioxins & -0.06 & -0.56 & 0.58 & 0.04 & 0.39 & 0.70 & $-\mathbf{0 . 1 9}$ & -2.03 & $0.045^{*}$ & -0.17 & -1.65 & 0.10 \\
\hline Total PCDD-TEQ ${ }^{\dagger}$ & -0.09 & -0.87 & 0.39 & 0.14 & 1.31 & 0.19 & -0.12 & -1.24 & 0.22 & -0.08 & -0.77 & 0.44 \\
\hline Total PCDF-TEQ ${ }^{\dagger}$ & -0.03 & -0.28 & 0.78 & 0.08 & 0.73 & 0.47 & -0.17 & -1.74 & 0.09 & -0.15 & -1.42 & 0.16 \\
\hline Total PCDD/PCDF-TEQ ${ }^{\dagger}$ & -0.08 & -0.75 & 0.45 & 0.13 & 1.19 & 0.24 & -0.14 & -1.39 & 0.17 & -0.10 & -0.95 & 0.34 \\
\hline Total non-ortho PCBs-TEQ $^{\dagger}$ & -0.03 & -0.28 & 0.78 & 0.01 & 0.08 & 0.94 & -0.16 & -1.67 & 0.10 & -0.22 & -2.04 & $0.04 *$ \\
\hline Total mono-ortho PCBs-TEQ ${ }^{\dagger}$ & -0.05 & -0.55 & 0.58 & 0.05 & 0.46 & 0.64 & -0.19 & -1.97 & 0.05 & -0.17 & -1.60 & 0.11 \\
\hline Total DL-PCB-TEQ ${ }^{\dagger}$ & -0.05 & -0.55 & 0.59 & 0.05 & 0.46 & 0.65 & -0.19 & -1.97 & 0.05 & -0.17 & -1.60 & 0.11 \\
\hline Total dioxins-TEQ $^{\dagger}$ & -0.05 & -0.53 & 0.60 & 0.09 & 0.84 & 0.41 & -0.15 & -1.52 & 0.13 & -0.15 & -1.39 & 0.17 \\
\hline
\end{tabular}

This table was constructed by reanalyzing the data from a previous study by Nakajima et al. [60]. Only statistically significant congeners are presented in this table $(* p<0.05)$

${ }^{\dagger}$ The TEQ levels were calculated by multiplying the levels of individual congeners by its TEF values of WHO 2005 [38]

allergies during the first 12 months of life. The characteristics of the participants and information pertaining to infant allergies were obtained from a baseline questionnaire administered to the mother during pregnancy, medical records from the time of delivery and a followup questionnaire when the child was 12 months of age. The risk of eczema, wheezing and food allergies during the first 12 months of life was not associated with maternal levels of 11 PFCs, including longer-chain compounds. Odds ratios for eczema and wheezing ranged from 0.66 to 0.73 and from 0.60 to 0.81 for the three higher quartiles of maternal PFTrDA levels, compared with the lowest in the adjusted models, but no doseresponse pattern was found [65]. 


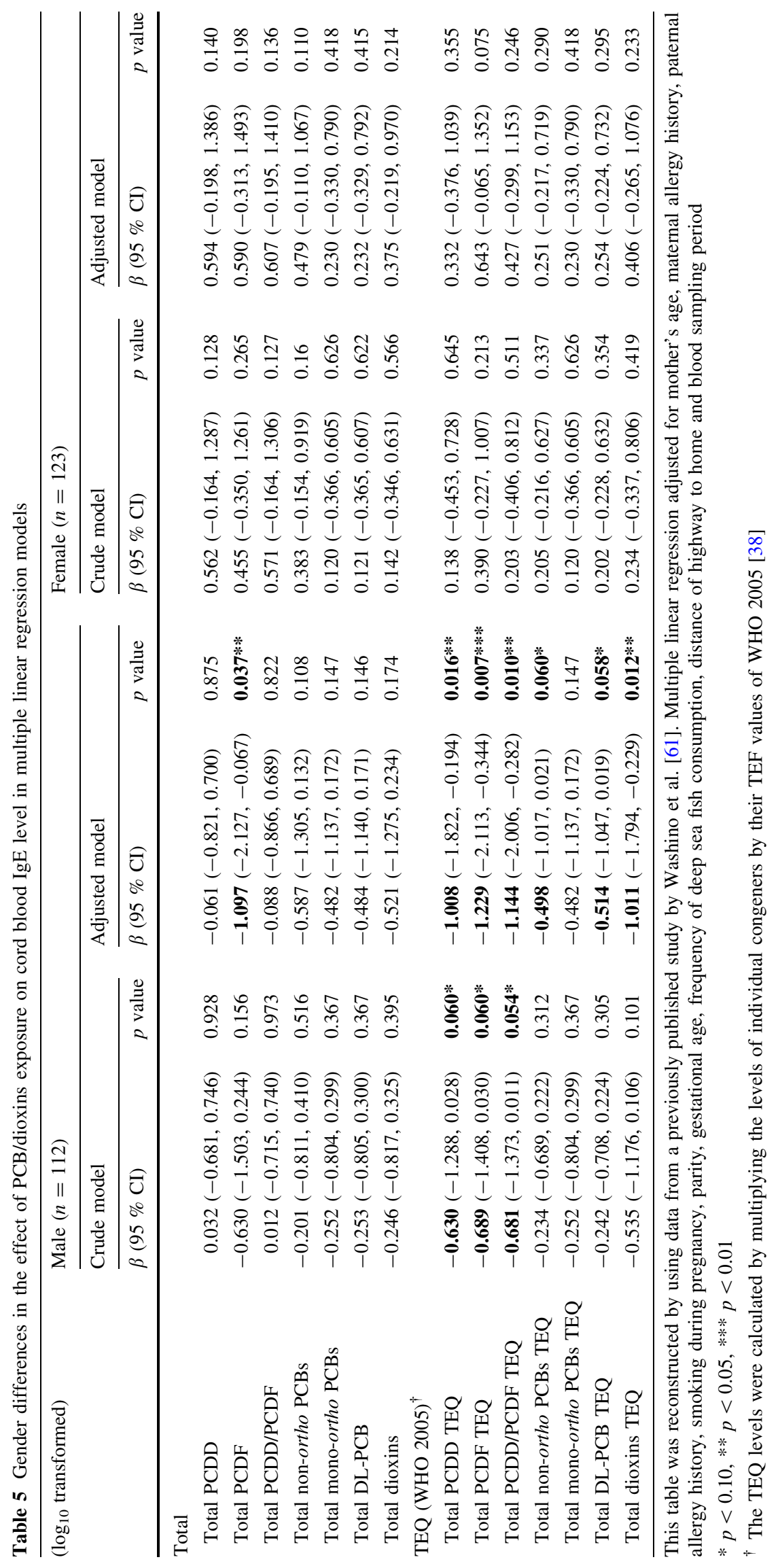


Table 6 Gender differences in the effect of PCB/dioxins exposure on the onset of otitis media at 18 months of age in multiple logistic regression models

\begin{tabular}{|c|c|c|c|c|c|}
\hline \multirow[t]{2}{*}{ ( $\log _{10}$ transformed) } & & \multicolumn{3}{|l|}{ Adjusted } & \multirow[t]{2}{*}{$p$-value for trenc } \\
\hline & & $\begin{array}{l}\text { Quartile } 2 \\
\text { OR (95 \% CI) }\end{array}$ & $\begin{array}{l}\text { Quartile } 3 \\
\text { OR (95\% CI) }\end{array}$ & $\begin{array}{l}\text { Quartile } 4 \\
\text { OR (95\% CI) }\end{array}$ & \\
\hline \multicolumn{6}{|l|}{ All } \\
\hline \multicolumn{6}{|l|}{ TEQ† } \\
\hline PCDDs & $\sum \mathrm{PCDDs}^{\dagger}$ & $1.2(0.53-2.7)$ & $1.1(0.50-2.6)$ & $1.5(0.65-3.5)$ & 0.39 \\
\hline PCDFs & $\sum \mathrm{PCDFs}^{\dagger}$ & $1.6(0.68-3.8)$ & $2.2(0.93-5.1)$ & $2.5(1.1-5.9)^{*}$ & $\mathbf{0 . 0 3}$ \\
\hline Non-ortho PCBs & $\sum$ Non-ortho $\mathrm{PCBs}^{\dagger}$ & $1.8(0.79-4.2)$ & $2.5(1.1-6.0)^{*}$ & $1.5(0.62-3.6)$ & 0.30 \\
\hline Total Dioxins $^{\dagger}$ & & $2.1(0.92-4.8)$ & $1.7(0.71-3.9)$ & $1.7(0.70-4.1)$ & 0.38 \\
\hline \multicolumn{6}{|l|}{ Congeners } \\
\hline PCDDs & OCDD & $3.4(1.4-8.5)^{*}$ & $2.8(1.1-7.0)^{*}$ & $2.6(1.0-6.9)^{*}$ & 0.12 \\
\hline PCDFs & $2,3,4,7,8-\mathrm{PeCDF}$ & $1.6(0.7-3.9)$ & $2.0(0.88-4.8)$ & $2.8(1.2-6.6)^{*}$ & 0.02 \\
\hline Non-ortho PCBs & $33^{\prime} 44^{\prime}-\mathrm{TCB}(\# 77)$ & $2.4(0.99-5.9)$ & $1.4(0.61-3.3)$ & $3.4(1.6-7.3)^{*}$ & 0.01 \\
\hline Mono-ortho PCBs & $233^{\prime} 44^{\prime} 5^{\prime}-\mathrm{HxCB}(\# 157)$ & $2.4(1.0-5.5)^{*}$ & $1.1(0.43-2.7)$ & $2.5(1.1-5.9)^{*}$ & 0.16 \\
\hline \multicolumn{6}{|l|}{ Males } \\
\hline \multicolumn{6}{|l|}{ TEQ $\dagger$} \\
\hline PCDDs & $\sum \operatorname{PCDDs}^{\dagger}$ & $0.5(0.13-1.8)$ & $2.0(0.65-6.2)$ & $2.9(0.83-10)$ & 0.03 \\
\hline PCDFs & $\sum$ PCDFs $\dagger$ & $1.0(0.28-3.3)$ & $2.9(0.87-9.8)$ & $3.8(1.1-13)^{*}$ & 0.01 \\
\hline Non-ortho PCBs & $\sum$ Non-ortho $\mathrm{PCBs}^{\dagger}$ & $2.4(0.70-8.3)$ & $2.9(0.86-9.7)$ & $3.6(0.98-13.3)$ & 0.05 \\
\hline Total dioxins & & $2.1(0.61-6.9)$ & $2.2(0.67-7.1)$ & $4.4(1.2-16)^{*}$ & $\mathbf{0 . 0 3}$ \\
\hline \multicolumn{6}{|l|}{ Congeners } \\
\hline PCDFs & 2,3,4,7,8-PeCDF & $1.7(0.48-6.0)$ & $2.9(0.87-10)$ & $5.3(1.5-19)^{*}$ & 0.01 \\
\hline \multirow[t]{2}{*}{ Non-ortho PCBs } & $33^{\prime} 44^{\prime}-\mathrm{TCB}(\# 77)$ & $2.8(0.85-9.4)$ & $0.9(0.24-3.4)$ & $3.5(1.2-11)^{*}$ & 0.08 \\
\hline & $33^{\prime} 44^{\prime} 55^{\prime}-\mathrm{HxCB}(\# 169)$ & $1.0(0.25-3.8)$ & $3.0(0.93-9.6)$ & $3.6(1.1-12)^{*}$ & 0.01 \\
\hline \multirow[t]{3}{*}{ Mono-ortho PCBs } & 2344'5-PeCB(\#114) & $2.4(0.62-8.9)$ & $4.5(1.2-16.6)^{*}$ & $4.9(1.3-18)^{*}$ & 0.01 \\
\hline & $23^{\prime} 44^{\prime} 55^{\prime}-\mathrm{HxCB}(\# 167)$ & $3.1(0.83-11)$ & $3.3(0.91-11)$ & $3.7(1.0-13)^{*}$ & 0.06 \\
\hline & $233^{\prime} 44^{\prime} 5^{\prime}-\mathrm{HxCB}(\# 157)$ & $4.5(1.2-17)^{*}$ & $1.6(0.37-6.5)$ & $7.5(1.9-29)^{*}$ & 0.02 \\
\hline \multicolumn{6}{|l|}{ Female } \\
\hline \multicolumn{6}{|l|}{ TEQ† } \\
\hline PCDDs & $\sum \operatorname{PCDDs}^{\dagger}$ & $2.3(0.71-7.6)$ & $0.5(0.11-2.0)$ & $1.1(0.30-4.1)$ & 0.44 \\
\hline PCDFs & $\sum \mathrm{PCDFs}^{\dagger}$ & $4.0(1.1-14.7) *$ & $1.2(0.30-5.1)$ & $1.3(0.29-5.8)$ & 0.41 \\
\hline Non-ortho PCBs & $\sum$ Non-ortho $\mathrm{PCBs}^{\dagger}$ & $1.3(0.41-4.3)$ & $1.9(0.51-7.1)$ & $0.8(0.22-3.1)$ & 0.86 \\
\hline Total Dioxins ${ }^{\dagger}$ & & $2.6(0.78-8.6)$ & $1.0(0.25-4.0)$ & $1.0(0.27-4.1)$ & 0.57 \\
\hline \multicolumn{6}{|l|}{ Congeners } \\
\hline Non-ortho PCBs & $33^{\prime} 44^{\prime}-\mathrm{TCB}(\# 77)$ & $1.4(0.3-6.9)$ & $1.5(0.45-4.9)$ & $3.8(1.2-12)^{*}$ & 0.03 \\
\hline
\end{tabular}

This table was reconstructed by using data from a previously published study by Miyashita et al. [62]. Only statistically significant congeners are presented in this table. $(* p<0.05)$. The OR $(95 \% \mathrm{CI})$ versus the first quartile (reference) in the logistic regression model was adjusted for maternal educational level, parity, infant gender, duration of breast-feeding, environmental tobacco exposure, day care attendance and blood sampling period (infant gender was excluded from covariates in gender-stratified analysis)

${ }^{a}$ quartiles applied as ordinal variables in the model

$* p<0.05, * * p<0.01$; Statistically significant, $p$-value

$\dagger$ The TEQ levels were calculated by multiplying the levels of individual congeners by its TEF values of WHO 2005 [38]

Gene-environment interaction

\section{The effects of maternal genetic polymorphisms on dioxin concentration}

Dioxins are metabolized by cytochrome P450, family 1 (CYP1) via AHR. We determined whether different blood dioxin concentrations are associated with polymorphisms in the $A H R$ (dbSNP ID: rs2066853), the $A H R R$ (rs2292596), the
CYPlA1 (rs4646903 and rs1048963), the CYPlA2 (rs762551) and the CYP1B1 (rs1056836) in pregnant Japanese women. Comparisons between the GG, GA and AA genotypes of the $A H R$ showed a significant difference for both the mono-ortho PCBs concentrations (genotype model; GG:GA:AA = 11,266.3:13,146.5:12,948.9 (pg/g lipid), $p=0.016)$ and that of toxicity equivalence quantities [TEQs] (GG:GA:AA $=0.338: 0.394: 0.388$ (TEQ pg/g lipid), $p=0.016$ ). Second, we found a significant 
association with the dominant genotype model for the PCDDs TEQs $([\mathrm{TT}+\mathrm{TC}): \mathrm{CC}=7.408: 6.480(\mathrm{TEQ} \mathrm{pg} / \mathrm{g}$ lipid), $p=0.048$ ) and for PCDFs TEQs ([TT + $\mathrm{TC}]: \mathrm{CC}=2.596: 2.267$ (TEQ pg/g lipid), $p=0.035$ ) of CYP1A1 (rs4646903). No significant differences were found among blood dioxin concentrations and polymorphisms in AHRR, CYP1A1 (rs1048963), CYP1A2 and CYP1B1. Thus, polymorphisms in $A H R$ and CYPlAl (rs4646903) were associated with maternal dioxin concentrations [55].

\section{Genetic polymorphisms and maternal smoking}

The effects of maternal smoking and genetic polymorphisms on infant birth size were examined in the Sapporo cohort. Birth weight and length were significantly lower among infants born to smokers with the AHR GG genotype, the CYPIA1 TC/CC genotype or the GSTM1 null genotype. When combinations of these genotypes were considered, birth weight and length were significantly lower for infants of continuously smoking women with the AHR GG genotype and CYP1A1 TC/CC genotype $(-315 \mathrm{~g}$ and $-1.7 \mathrm{~cm}$, respectively) and with the CYPIAI TC/CC genotype and GSTM1 null genotype ( $-237 \mathrm{~g}$ and $-1.3 \mathrm{~cm}$, respectively) [56] (Fig. 4). For polymorphisms in the gene-encoding $\mathrm{N}$-nitrosamine-metabolizing enzymes, $\mathrm{NQO1}$, birth weight, birth length and birth head circumference were significantly reduced $(-199 \mathrm{~g},-0.8 \mathrm{~cm}$ and $-0.7 \mathrm{~cm}$, respectively) among infants born to smokers with the $N Q O 1 \mathrm{CC}$ genotype (Fig. 5). This genotype did not confer adverse effects among women who had never smoked or who quit smoking during the first trimester. Our results suggest an

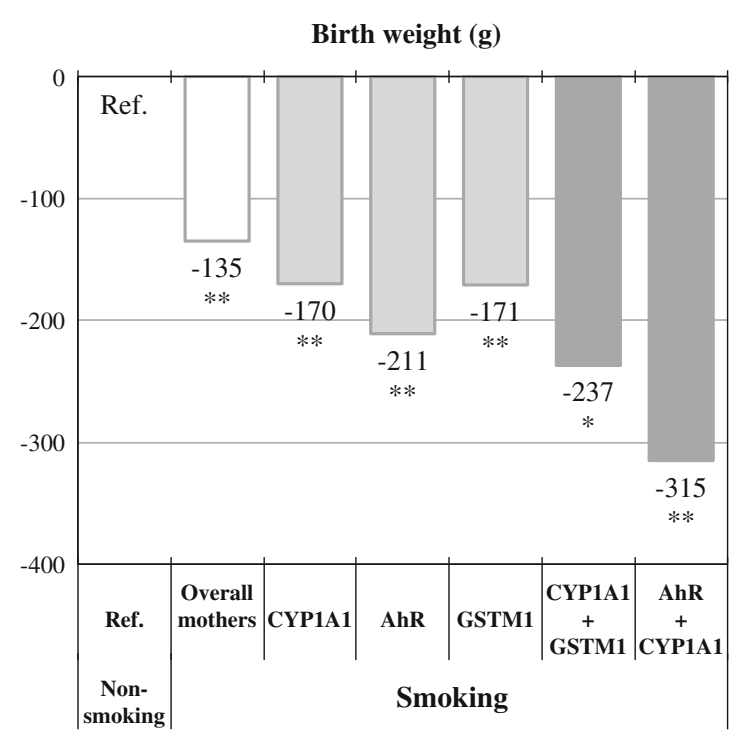

Fig. 4 The effects of maternal smoking in combination with maternal PAHs-metabolism-related genetic polymorphisms on infants' birth size. Adjusted for maternal age, height, weight before pregnancy, alcohol consumption during pregnancy, history of delivery, newborn important modifying role of polymorphisms in metabolizing enzyme genes in concert with the adverse effects of maternal smoking on infant birth size [57].

\section{Folate, maternal smoking and genetic polymorphisms}

Folate is essential for fetal growth and development, and smoking has been associated with nutritional deficiencies in vitamins including folate. The birth weight of infants born to moderate smokers ( $\geq 10$ cigarettes per day) with low folate status $(<6.0 \mathrm{ng} / \mathrm{ml})$ was lower by $107 \mathrm{~g}$ compared with non-smokers having a normal folate status $(\geq 6.0 \mathrm{ng} / \mathrm{ml})$. Maternal 5,10-methylenetetrahydrofolate reductase (MTHFR) 1298AA was associated with low folate status. The 5,10-MTHFR AA genotype was associated with a decrease in birth weight by $107 \mathrm{~g}$ in infants born to smokers. After stratification by infant gender, the effect was more pronounced in male infants with a reduction in birth weight of $117 \mathrm{~g}$. Female infants never demonstrated any statistically significant changes in birth weight [58].

\section{Discussion}

What are the primary strengths and weaknesses of the study?

The design of our study is a prospective cohort study intended to collect data on environmental exposures during fetal development and to control for potential confounders.

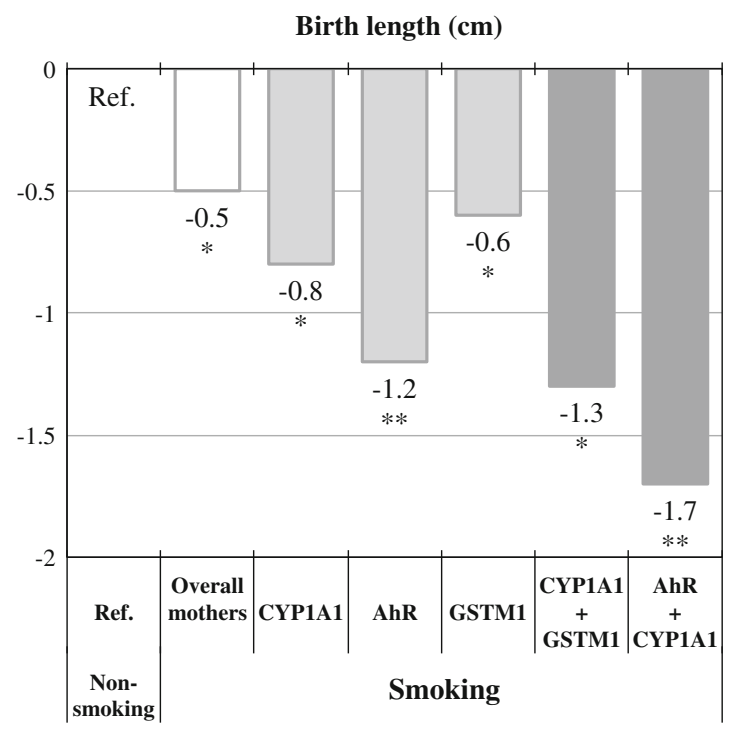

sex, gestational weeks and household income. $* p<0.05$, $* * p<0.01$ This figure was created by modifying a figure contained in our previous study by Sasaki et al. [56] 


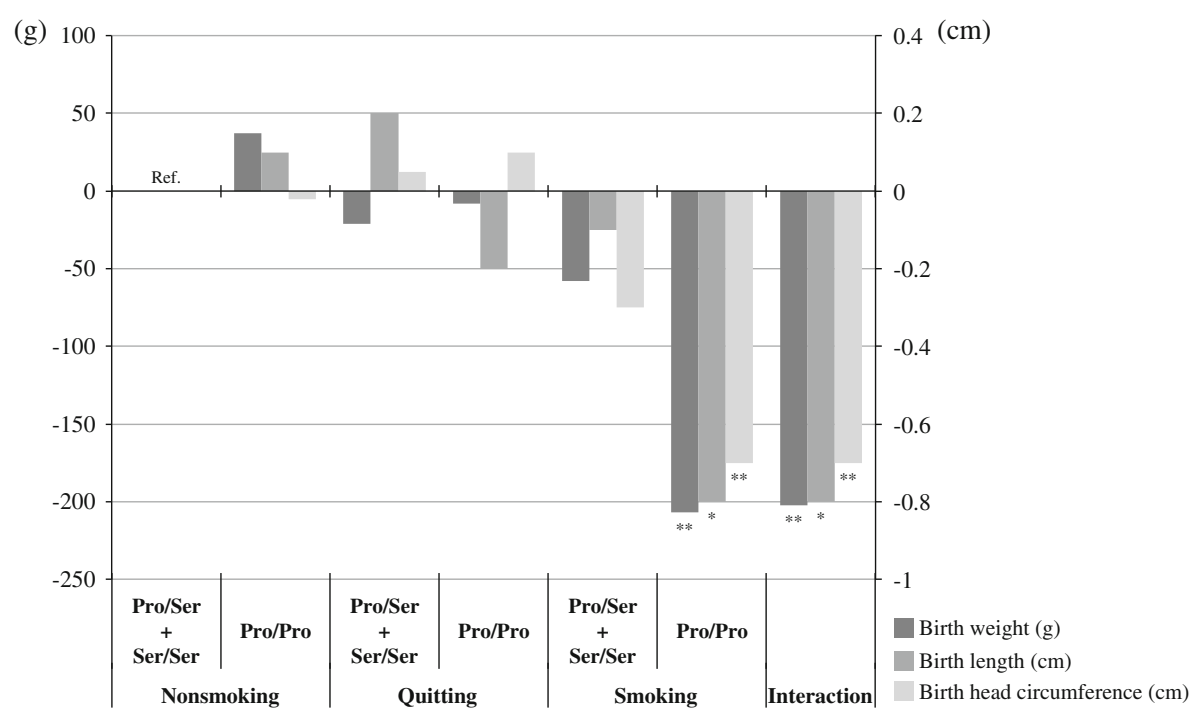

Fig. 5 The effects of maternal smoking in combination with maternal NQO1 genotype on infants' birth size. Adjusted for maternal age, height, weight before pregnancy, weight gain during pregnancy, alcohol consumption during pregnancy, parity, infant gender, gestational age, and household income. Interaction in multiple linear regression models was defined as product terms for the product of the

The detailed measurements in exposures and outcomes are adequate to detect the various effects of perinatal environmental and genetic determinants on childhood outcomes. In the Sapporo cohort study, face-to-face examinations for neurodevelopment assessment were conducted. The Hokkaido cohort had been the largest birth cohort in Japan until 2011 when the nation-wide cohort study, the Japan Environment and Children's Study (JECS), was launched based upon our study design. A potential problem of our study is that both the Sapporo and Hokkaido cohorts may have been biased in participant selection because they are both hospital-based studies, although the latter cohort consists of the hospitals and clinics over the Hokkaido areas to mitigate that bias (Fig. 1). In addition, despite our efforts to keep track of participants' residence with periodical newsletters, some levels of attrition were caused by individuals moving outside of the study area.

The main findings of the study

Over the last decade, we have been intensely investigating the effects of intrauterine chemical exposures on children's health. The main findings of our study are as follows.

1. The effects of dioxins-with emphasis on gender differences

We discovered that there are gender differences in the effects of dioxins and DL-PCBs on birth weight, infants' neurodevelopment and immune functions; our results dummy independent variables: maternal smoking status (nonsmoker, quitter or smoker) and genotype (wild or mutant). $\beta$ represents the product term for smoker $\times$ wild genotype. $N Q O 1 \mathrm{NAD}(\mathrm{P}) \mathrm{H}$ : quinone oxidoreductase 1, Pro proline, Ser serine This figure was constructed by using the data from previous study by Sasaki et al. [57]

suggest that the male infants are more susceptible to those chemicals than female infants. Our observations on birth weight were in concordance with other studies, which indicated a stronger negative effect of these compounds on the birth weight of male infants [66-70]. In our study, we found that the adjusted regression coefficients of total PCDDs TEQ and PCDDs/PCDFs TEQ levels among male and female infants were -331.4 and $-126.3 \mathrm{~g}$ and -338.7 and $-173.9 \mathrm{~g}$, respectively. It is possible that male infants had lower birth weights at higher PCDDs and PCDDs/ PCDFs TEQ levels in the maternal blood than female infants. In addition to birth weight, we also found that dioxin-like compounds had negative effects on neurodevelopment at 6 months of age in addition to the negative effects on infants' immune function such as cord blood IgE levels and otitis media at 18 months of age. Although there are few epidemiological studies examining the effects of intrauterine exposure to dioxin-like compounds that specifically examined gender differences other than birth weight, it appears that male infants are more susceptible to exposure to these chemicals, which might be due to gender-specific endocrine activities. However, examining gender difference in the effects of PCBs and PCDDs/ PCDFs are part of a larger discussion on endocrine disruption; therefore, we need more evidence from larger studies with exposure measurements. Recently, we analyzed sex hormone concentrations in cord blood and its correlation with intrauterine EDCs exposure. In the near future, we will be able to examine gender-specific responses to EDCs and their effect on sex hormone levels. 
In addition to further epidemiological studies, molecular biological studies using animal models and human cell lines are also necessary to elucidate the molecular mechanisms of gender-dependent susceptibility to the exposures.

2. The different effects of dioxin congeners

We discovered that the different dioxin congeners had different effects on children exposed in utero. Identification of the potent biological properties of PCDDs, PCDFs and DL-PCBs, and which individual congeners of PCDDs, PCDFs and DL-PCBs affect birth outcomes has been an important goal in investigating the mechanism of effect to prevent harmful effects on fetuses. We found negative associations between maternal PCDF and PCDD exposure levels and birth weight and motor development at 6 months of age, and an increased risk of developing otitis media at 18 months of age correlated with maternal PCDF exposure.

In the study of Yu-Cheng children, it was indicated that the PCDFs group, including the penta-CDF and hexa-CDF congeners, were primarily responsible for the observed health effects compared to other groups of $\mathrm{PCBs} / \mathrm{PCDFs}$ congeners [71]. Moreover, $70 \%$ of the toxicity of TEQ was contributed by $2,3,4,7,8-\mathrm{PeCDF}$ in Yusho patients [72]. These observations were in concordance with our results, which indicated a significant negative association between 2,3,4,7,8-PeCDF and birth weight. In addition, we found that maternal 2,3,4,7,8-PeCDF exposure increased the risk of developing otitis media at 18 months of age. These data suggest that $2,3,4,7,8-\mathrm{PeCDF}$ is one of the most dangerous congeners.

Due to its high affinity for the AHR, it was suggested that there is a specific accumulation of PCDDs and PCDFs congeners including 2,3,4,7,8-PeCDF in the placenta [73, 74], which plays an important role in transporting nutrients and oxygen through cord blood in the developing fetus. Taking the above considerations into account, we suggest that PCDDs and PCDFs congeners, especially 2,3,4,7,8PeCDF, may accumulate in the placenta and retard important placental functions, which may result in lower birth weight.

We also found significant negative associations between motor development and maternal exposure to isomers of PCDDs and PCDFs and mental development and exposure to levels of total PCDDs and PCDFs. Currently, there were few human or animal experimental studies that have investigated the association between individual isomer levels of PCBs and dioxins and neurodevelopment. These studies are required to elucidate the mechanisms of action of individual congeners on neurodevelopment.

\section{The diverse effects of PFCs exposure}

Our results suggest that intrauterine PFCs exposure affects not only fetal growth but also the immune system.
In the current study, cord blood IgE levels decreased significantly with high maternal PFOA concentrations in female infants. However, no association was observed between maternal serum PFOS and PFOA concentrations and the occurrence of food allergies, eczema, wheezing and otitis media in their infants during the first 18 months of life. The results of the C8 Health Project showed a significant trend in decreasing $\operatorname{IgE}$ levels with increasing PFOA levels in maternal blood samples among females [75]. Our results are consistent with those of that study, even though the concentration of maternal PFOA was lower than that measured in other studies, including the $\mathrm{C} 8$ Health Project [75-78]. However, we note that the PFOA levels were not associated with the development of allergies and infectious diseases in infants before 18 months of age. In addition, our result contradicted the results of the Taiwan study, which showed that PFOA levels were positively correlated with cord blood IgE levels only in males [21]. It may be necessary to perform follow-up studies to investigate whether prenatal exposure to PFCs affects immune system development (and address potential gender-specific differences) from infancy to school age because it is difficult to obtain definitive diagnoses for infants.

Moreover, a recent result from a prospective cohort study suggested that intrauterine exposure to PFCs could also modulate infants' thyroid hormone levels [19]. They reported that there were significant negative correlations between maternal PFOS and fetal T3, and maternal PFTrDA and fetal T4 and T3 after adjusting for major covariates. However, this was the only epidemiological report regarding prenatal PFCs exposure and infants' thyroid function, and their sample size was insufficient. Thus, we need additional epidemiological studies to validate the effects of intrauterine PFCs exposure on thyroid functions.

In addition, the temporal trends of PFCs levels indicates that PFOS and PFOA concentrations were decreasing every year from 2003 to 2011 due to the restriction of PFOS by the Stockholm Convention on Persistent Organic Pollutants in 2009. Instead, PFNA and PFDA, which have a longer carbon chain than PFOA and are harder to be metabolized in the body, were increasing. Further studies must be conducted to estimate the effects of intrauterine exposure to long-chained PFCs on children's health and development.

\section{Genetic susceptibility to the exposures}

In our study, we found that the maternal genetic polymorphisms in $A H R$ or CYPIAl independently modified dioxin concentrations in maternal blood, suggesting different dioxin accumulation in the body of individuals with these genotypes, which would lead to different dioxin exposure levels [55]. CYP1Al activation mediated by AHR 
is an important mechanism for metabolizing dioxins. Dioxins such as 2,3,7,8-tetrachlorodibenzo- $p$-dioxin (TCDD) are sensitive to AHR, and TCDD mediates transcriptional regulation of AHR via its binding with AHR nuclear translocator. Activated AHR facilitates the expression of CYP1A1, CYP1A2, CYP1B1 and AHRR, which are important for metabolizing dioxins [79]. Moreover, the expression of CYPIAI and CYPIBI are important for endocrine signaling pathways. Those proteins mediate the transformation of $17 \beta$-estradiol $\left(E_{2}\right)$ /estrone $\left(E_{1}\right)$ to the biologically active metabolites 2-hydroxyestradiol $\left(2-\mathrm{OH}-\mathrm{E}_{2}\right)$ and 4-hydroxyestradiol (4-OH-E 2 ) [80].

In addition to the dioxin concentrations, among the polymorphism groups of CYPIAl, AHR, GSTM1 and $N Q O 1$, we observed different susceptibilities with respect to the effect of maternal smoking exposure on birth size $[56,57]$. The AHR, CYP1A1 and GSTM1 metabolize the polycyclic aromatic hydrocarbon (PAH) in tobacco smoke. The GSTM1 detoxifies specific biologically active metabolites of PAHs, and carriers of the GSTM1 null genotype have a reduced ability to detoxify these metabolites. Our study shows that infants born to mothers that have the $A H R$ wild genotype and continuously smoke had a significantly lower birth weight and length compared with infants born to non-smokers; moreover, smokers who had the $A H R$ wild type and CYPlAl variant genotype had the greatest reduction in both birth weight and length. Because there have only been a few epidemiological studies, further studies are required to clarify the role of the Arg554Lys polymorphism in fetal development.

The NQO1 is an important enzyme that functions in both phase I (activation) and phase II (detoxification) metabolism of xenobiotics depending on the substrate. As a detoxification enzyme, it catalyzes the two-electron reduction of quinoid compounds to the readily excreted hydroquinones to prevent the generation of reactive oxygen species and, thereby, protect cells against oxidative damage. It also catalyzes the activation of some pro-carcinogens such as nitrosamines and heterocyclic amines, which are present in tobacco smoke [81]. Our study suggests an important role for polymorphisms in the $N$-nitrosaminemetabolizing enzyme gene $\mathrm{NQO}$ in mitigating the adverse effects of maternal smoking on infant birth size. These findings could have significant public health implications regarding the need for smoking prevention and cessation programs aimed specifically at susceptible women of childbearing age.

In addition, our current results suggest that the adverse health effects of prenatal tobacco smoke exposure resulted not only from active smoking but also from secondhand smoke (SHS) exposure during pregnancy. Birth weight and infant length among SHS-exposed women with the CYP1A1*2C AG/GG genotypes $(-88 \mathrm{~g}$ and $-0.9 \mathrm{~cm}$, respectively) and the epoxide hydrolase $1(E P H X I) \mathrm{His} / \mathrm{His}$ genotypes $(-154 \mathrm{~g}$ and $-1.1 \mathrm{~cm}$, respectively) were significantly lower. The $\mathrm{N}$-acetyl transferase $2(N A T 2 * 7)$ slow acetylators group was also adversely affected $(-51 \mathrm{~g})$. A combination of EPHXI His/His $+N A T 2 * 7$ slow alleles not only resulted in a remarkable decrease in birth weight and length $(-145 \mathrm{~g}$ and $-1.1 \mathrm{~cm}$, respectively) but also demonstrated significant interaction with SHS exposure [82].

The future challenges of the study

1. Inferences from previous studies in hypospadiasgene-environment interactions

As described in our previous review, both genetic and environmental factors contribute to the etiology of congenital malformations such as hypospadias and cryptorchidism [26]. The etiology of hypospadias was unclear in a majority of cases, but it was regarded as a complex disorder caused by both genetic and environmental factors (Fig. 6). Because the development of the urethral and external genital system in the male fetus is androgendependent, abnormalities in the synthesis and metabolism of androgens caused by exposure to EDCs can result in abnormal genital developmental phenotypes.

In previous studies, we had clarified the etiology of hypospadias with genetic factors that were related to fetal endocrine activity such as the ESRI and ESR2 and $17 \beta H S D 3$ and maternal hormonal activity such as the CYP1A1 in a retrospective case-control study [23, 24, 26]. Hypospadias is a common congenital anomaly caused by an incomplete fusion of the urethral folds. The urethral opening is on the ventral surface of the penis, on the scrotum or the perineum. Thus far, an increase in the prevalence of hypospadias has been reported in various countries, and these trends are speculated to be related to EDC exposure [83]. Several studies have shown the association between hypospadias and fetal gene polymorphisms in genes involved in androgen metabolism [84-86].

These results suggest that environmental factors, including EDCs exposure in utero, as well as genetic factors are responsible for the etiologies of congenital malformations, diseases and birth outcomes such as birth size. Moreover, considering that environmental exposures in utero might affect the children's birth outcomes, the mother's EDCs exposure level and genetic factors that may affect the intrauterine environment are also important factors to consider in evaluating the cause of adverse birth outcomes. Thus, to elucidate the etiology of the disease, two different study approaches must be conducted; one is the screening for genetic risk factors in children and mothers, and the other is to estimate the effect of the environmental risk factors including EDC exposures. In 


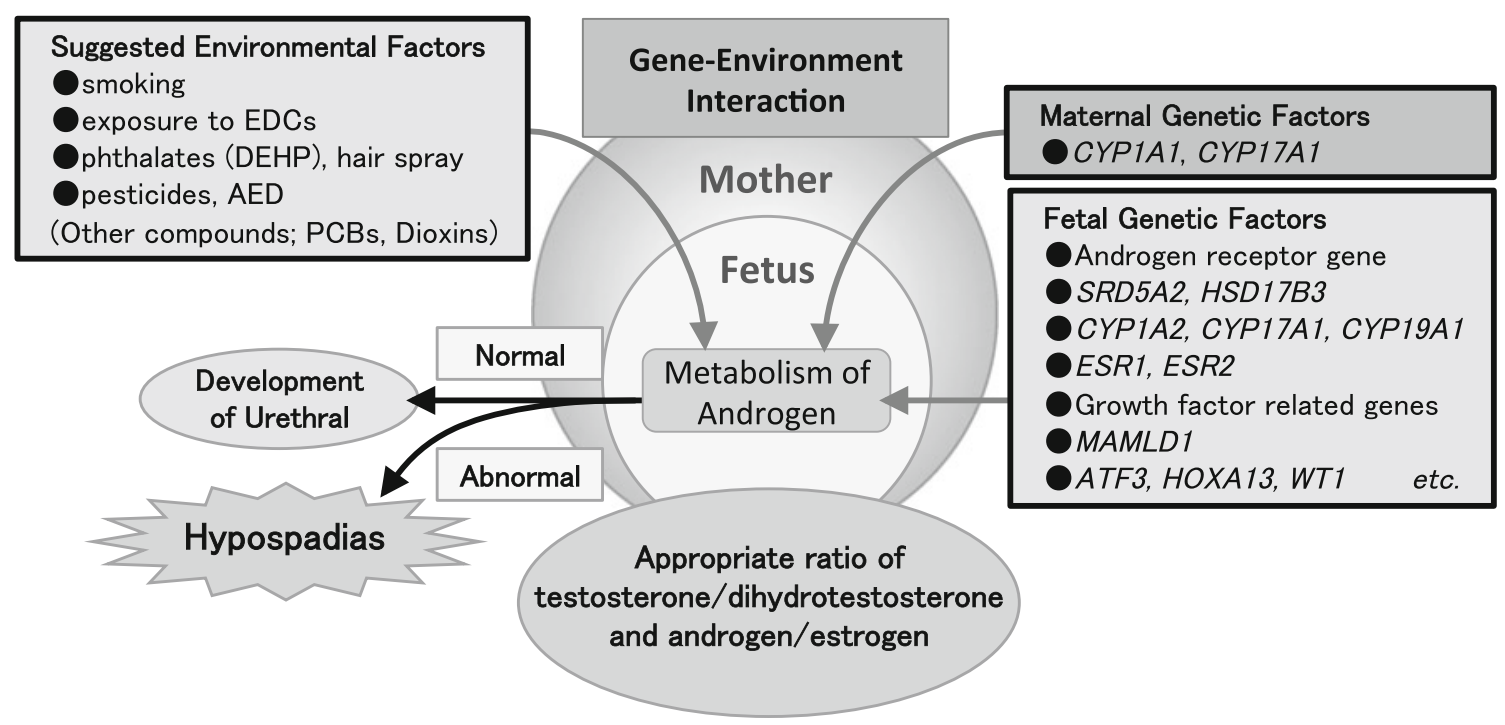

Fig. 6 Summary and suggestions for further studies on the environmental and genetic factors that influence hypospadias development. This figure was created by modifying a figure contained in our previous review by Kishi et al. [26]

addition, by integrating those two approaches to study gene-environment interaction, it becomes possible to identify more susceptible individuals in the population.

2. Gene-environment interactions involved in the etiology of ADHD

In recent years, the increased prevalence of developmental disorders such as Autism Spectrum Disorder (ASD) and ADHD are of increasing concern to the public. Although it is estimated that genetic effects account for $80 \%$ of ASD cases and $79 \%$ of ADHD cases, respectively [87], environmental factors such as the nursing environment and exposure to tobacco smoke also appear to be important factors because the prevalence of these diseases continues to increase while the genetic background of the population remains relatively stable. To date, postnatal environmental exposures, such as passive smoke exposure, iron deficiency, thyroid dysfunction, otitis media and psychosocial stress, are reported as risk factors for ADHD. In addition, prenatal risk factors such as maternal smoking, maternal alcohol intake, lead, PCBs and food additive exposure are also reported to be risk factors for ADHD [88]. Additionally, several studies have indicated that children who were born prematurely or with low birth weight had an increased risk of developing ADHD [8992], which suggests that the intrauterine environment may play some role. However, the detailed mechanisms of the etiology of those neurodevelopmental disorders have yet to be identified. In the future, by taking genetic and environmental study approaches and studying gene-environment interactions, it is anticipated that all possible risk factors will be elucidated, and eventually, the etiologies of developmental disorders such as Autism and ADHD will be known.

In the present cohort studies, we discovered the maternal genetic factors that affect a child's birth outcome along with the risks associated with maternal smoking and intrauterine dioxin exposure. However, there are few genetic risk factors that have been found thus far considering the large, intricate gene networks involved in a child's health and development. Further studies including genome wide analysis are needed to elucidate the effects of gene-environment interactions.

\section{The role of epigenetics}

Recently, there has been a growing interest in understanding the role of epigenetics in linking a child's intrauterine environment to future health and disease. Epigenetic modifications, such as DNA methylation, are programmed in utero and are likely to be maintained through cell division and throughout cell linages [93]. Therefore, it is postulated that epigenetic regulation is the "missing link" in the DOHaD hypothesis, which would connect the intrauterine environment to postnatal phenotypes. To date, dozens of animal studies and several epidemiological studies have been conducted to estimate the effect of maternal smoking, environmental chemical exposure and metal exposure in utero on a child's epigenome [94-96]. For instance, maternal smoking exposure increases the methylation of the regulatory region of Insulin-like Growth Factor 2 (IGF2) in cord blood DNA, which negatively correlates with IGF2 protein levels in the cord blood [97, 98]. However, at this moment, the epigenetic effects of intrauterine exposure to environmental chemicals are controversial. Further studies 
exploring the environmental and genetic risk factors for epigenetic vulnerability is necessary. Currently, we are conducting epigenetic research to investigate the effect of intrauterine exposures to environmental chemicals on a child's epigenome and the resulting risk for future health and disease complications.

As Barker first suggested, the consequences of a disrupted intrauterine environment might be expressed as adverse health outcomes a decade more or later. To thoroughly estimate the effects of intrauterine EDCs exposures in humans, it is necessary to follow individuals in a prospective birth cohort study with a sufficient sample size for a long period.

\section{Working toward international collaboration}

In recent years, there has been an avid movement toward collaborating and integrating existing birth cohort studies across borders. The primary purpose of these birth cohort consortiums are to obtain evidence based results by using data from larger sample sizes (meta-analysis), as well as obtaining more applicable and generalizable results by integrating data beyond regions, countries and ethnicities. For instance, in Europe, the Environmental Health Risks in European Birth Cohorts (ENRIECO) was established in 2009 [99]. In Asia, the Birth Cohort Consortium of Asia (BiCCA) is now calling for participation to all existing Asian birth cohorts [http://www.bicca.org]. Although there are many challenges regarding coordination of different cohort studies, we do believe that it is a worthy endeavor.

Additional information concerning the Hokkaido study is available at the study website: http://www.cehs.hokudai. ac.jp/. All of the source data that have been collected are maintained and stored at Hokkaido University Center for Environmental and Health Sciences. Initial approaches or enquiries regarding the study can be made to the principal investigator (rkishi@med.hokudai.ac.jp).

Acknowledgments We would like to express our appreciation to all of the study participants of The Hokkaido Study on Environment and Children's Health. We express our profound gratitude to all personnel in the hospitals and clinics that collaborated with the study: Sapporo Toho Hospital, Keiai Hospital, Endo Kikyo Maternity Clinic, Shiroishi Hospital, Memuro Municipal Hospital, Aoba Ladies Clinic, Obihiro-Kyokai Hospital, Akiyama Memorial Hospital, Sapporo Medical University Hospital, Hokkaido University Hospital, Kitami Red Cross Hospital, Hoyukai Sapporo Hospital, Gorinbashi Hospital, Hashimoto Clinic, Asahikawa Medical College Hospital, Hakodate Central General Hospital, Ohji General Hospital, Nakashibetsu Municipal Hospital, Sapporo Tokushukai Hospital, Asahikawa Red Cross Hospital, Wakkanai City Hospital, Kushiro Rosai Hospital, Sapporo-Kosei General Hospital, Shibetsu City General Hospital, Nikko Memorial Hospital, Sapporo City General Hospital, Kohnan Hospital, Hakodate City Hospital, Hokkaido Monbetsu Hospital, Tenshi Hospital, Hakodate Goryoukaku Hospital, Nakamura Hospital, Kin-ikyo Sapporo Hospital, Kitami Lady's Clinic, Engaru-Kosei
General Hospital, Kushiro Red Cross Hospital, Nayoro City General Hospital and Obihiro-Kosei General Hospital. We also deeply express our gratitude to all of the staff of The Hokkaido Study on Environment and Children's Health for their considerable efforts to support our study: Y. Tanabe, M. Kato, Y. Okada, K. Tanaka, M. Hoshiba, N. Takeshima and M. Kihara. This study was funded by a Grant-in-Aid for Scientific Research from the Japanese Ministry of Health, Labor and Welfare; the Ministry of Education, Culture, Sports, Science and Technology; the Japan Society for the Promotion of Science; and the Ministry of Environment.

Conflict of interest None declared.

\section{Appendix: Members of The Hokkaido Study on Environment and Children's Health}

S. Tajima, H. Goudarzi, K. Azumi, A. Kanazawa, Y. Otake, T. A. Yila (Hokkaido University Center for Environmental and Health Sciences, Sapporo, Japan), Y. Ait Bamai, S. Cong, Tos. Baba, T. S. Braimoh, S. Ban, N. Washino, K. Konishi, S. Kato, A. Uno, M. Limpar (Department of Public Health Sciences, Hokkaido University Graduate School of Medicine, Sapporo), H. Minakami (Department of Obstetrics and Gynecology, Hokkaido University Graduate School of Medicine, Sapporo), K. Nonomura (Department of Renal and Genitourinary Surgery, Hokkaido University Graduate School of Medicine, Sapporo), T. Mitsui (Department of Urology, Hokkaido University Graduate School of Medicine, Sapporo), T. Endo, Tsu. Baba (Sapporo Medical University, Sapporo), K. Sengoku, Y. Saijo, E. Yoshioka, T. Miyamoto (Asahikawa Medical University, Asahikawa), M. Yuasa (Juntendo University, Tokyo), F. Sata (Department of Epidemiology, National Institute of Public Health, Wako), N. Kurahashi (Epidemiology and Prevention Division, Research Center for Cancer Prevention and Screening, National Cancer Center, Tokyo), J. Tamaki (School of Medicine, Kinki University), J. Kajiwara, T. Todaka (Fukuoka Prefectural Institute of Health and Environmental Sciences, Fukuoka), H. Murohashi (Graduate School of Education, Hokkaido University, Sapporo), H. Matsuura (Laboratory of Bioorganic Chemistry, Division of Applied Bioscience, Research Faculty of Agriculture, Hokkaido University, Sapporo), T. Matsumura (IDEA Consultants,Inc., Shizuoka), M. Ishizuka (Laboratory of Toxicology, Graduate School of Veterinary Medicine, Hokkaido University, Sapporo).

\section{Collaborating Institutions}

Hokkaido University Center for Environmental and Health Sciences; Hokkaido University Graduate School of Medicine: Departments of Public Health Sciences, Obstetrics and 
Gynecology, Pediatrics, Renal and Genitourinary Surgery, Respiratory Medicine and Dermatology; Hokkaido University Graduate School of Veterinary Medicine: Department of Environmental Veterinary Sciences; Hokkaido University Graduate School of Agricultures; Sapporo Medical University: Obstetrics and Gynecology; Asahikawa Medical College: Department of Health Sciences, Obstetrics and Gynecology; Sapporo City Institute of Public Health; Hokkaido Association of Obstetricians and Gynecologists; Fukuoka Institute of Health and Environmental Sciences; Hoshi University School of Pharmacy and Pharmaceutical Sciences, Department of Analytical Chemistry; IDEA Consultants, Inc., Sizuoka; Chubu University, Nagoya.

\section{References}

1. Colborn T, Dumanoski D, Myers JP. Our stolen future: are we threatening our fertility, intelligence, and survival? A scientific detective story. New York: Plume; 1997.

2. Wigle DT, Arbuckle TE, Turner MC, Berube A, Yang Q, Liu S, et al. Epidemiologic evidence of relationships between reproductive and child health outcomes and environmental chemical contaminants. J Toxicol Environ Health B Crit Rev. 2008; 11(5-6):373-517.

3. Schug TT, Janesick A, Blumberg B, Heindel JJ. Endocrine disrupting chemicals and disease susceptibility. J Steroid Biochem Mol Biol. 2011;127(3-5):204-15.

4. Meeker JD. Exposure to environmental endocrine disruptors and child development. Arch Pediatr Adolesc Med. 2012;166(6):E1-7.

5. Barker DJ, Osmond C. Infant mortality, childhood nutrition, and ischaemic heart disease in England and Wales. Lancet. 1986;1(8489):1077-81.

6. Gluckman P, Hanson M. Developmental origins of health and disease. New York: Cambridge University Press; 2006.

7. Newman J, Ross M. Early life origins of human health and disease. Basel: Karger; 2009.

8. Dietert RR. Developmental immunotoxicology: focus on health risks. Chem Res Toxicol. 2009;22(1):17-23.

9. El Majidi N, Bouchard M, Gosselin NH, Carrier G. Relationship between prenatal exposure to polychlorinated biphenyls and birth weight: a systematic analysis of published epidemiological studies through a standardization of biomonitoring data. Regul Toxicol Pharmacol. 2012;64(1):161-76.

10. Boucher O, Muckle G, Bastien CH. Prenatal exposure to polychlorinated biphenyls: a neuropsychologic analysis. Environ Health Perspect. 2009;117(1):7-16.

11. Goodman JE, Kerper LE, Boyce CP, Prueitt RL, Rhomberg LR. Weight-of-evidence analysis of human exposures to dioxins and dioxin-like compounds and associations with thyroid hormone levels during early development. Regul Toxicol Pharmacol. 2010;58(1):79-99.

12. Vreugdenhil HJ, Slijper FM, Mulder PG, Weisglas-Kuperus N. Effects of perinatal exposure to PCBs and dioxins on play behavior in Dutch children at school age. Environ Health Perspect. 2002;110(10):A593-8.

13. Su PH, Huang PC, Lin CY, Ying TH, Chen JY, Wang SL. The effect of in utero exposure to dioxins and polychlorinated biphenyls on reproductive development in eight year-old children. Environ Int. 2012;39(1):181-7.
14. Winans B, Humble MC, Lawrence BP. Environmental toxicants and the developing immune system: a missing link in the global battle against infectious disease? Reprod Toxicol. 2011;31(3): 327-36.

15. Apelberg BJ, Witter FR, Herbstman JB, Calafat AM, Halden RU, Needham LL, et al. Cord serum concentrations of perfluorooctane sulfonate (PFOS) and perfluorooctanoate (PFOA) in relation to weight and size at birth. Environ Health Perspect. 2007;115(11): 1670-6.

16. Fei C, McLaughlin JK, Tarone RE, Olsen J. Perfluorinated chemicals and fetal growth: a study within the Danish National Birth Cohort. Environ Health Perspect. 2007;115(11):1677-82.

17. Olsen GW, Butenhoff JL, Zobel LR. Perfluoroalkyl chemicals and human fetal development: an epidemiologic review with clinical and toxicological perspectives. Reprod Toxicol. 2009; 27(3-4):212-30.

18. Halldorsson TI, Rytter D, Haug LS, Bech BH, Danielsen I, Becher G, et al. Prenatal exposure to perfluorooctanoate and risk of overweight at 20 years of age: a prospective cohort study. Environ Health Perspect. 2012;120(5):668-73.

19. Kim S, Choi K, Ji K, Seo J, Kho Y, Park J, et al. Trans-placental transfer of thirteen perfluorinated compounds and relations with fetal thyroid hormones. Environ Sci Technol. 2011;45(17): 7465-72.

20. Grandjean P, Andersen EW, Budtz-Jorgensen E, Nielsen F, Molbak K, Weihe $\mathrm{P}$, et al. Serum vaccine antibody concentrations in children exposed to perfluorinated compounds. JAMA. 2012;307(4):391-7.

21. Wang IJ, Hsieh WS, Chen CY, Fletcher T, Lien GW, Chiang HL, et al. The effect of prenatal perfluorinated chemicals exposures on pediatric atopy. Environ Res. 2011;111(6):785-91.

22. World Health Organization. Possible developmental early effects of endocrine disrupters on child health. Geneva: WHO Press; 2012.

23. Ban S, Sata F, Kurahashi N, Kasai S, Moriya K, Kakizaki H, et al. Genetic polymorphisms of ESR1 and ESR2 that may influence estrogen activity and the risk of hypospadias. Hum Reprod. 2008;23(6):1466-71.

24. Sata F, Kurahashi N, Ban S, Moriya K, Tanaka KD, Ishizuka M, et al. Genetic polymorphisms of 17 beta-hydroxysteroid dehydrogenase 3 and the risk of hypospadias. J Sex Med. 2010;7(8): 2729-38.

25. Kurahashi N, Sata F, Kasai S, Shibata T, Moriya K, Yamada H, et al. Maternal genetic polymorphisms in CYP1A1, GSTM1 and GSTT1 and the risk of hypospadias. Mol Hum Reprod. 2005; 11(2):93-8.

26. Kishi R, Sata F, Yoshioka E, Ban S, Sasaki S, Konishi K, et al. Exploiting gene-environment interaction to detect adverse health effects of environmental chemicals on the next generation. Basic Clin Pharmacol Toxicol. 2008;102(2):191-203.

27. Kishi R, Sasaki S, Yoshioka E, Yuasa M, Sata F, Saijo Y, et al. Cohort profile: the Hokkaido study on environment and children's health in Japan. Int J Epidemiol. 2011;40(3):611-8.

28. The International Study of Asthma and Allergies in Childhood (ISAAC) Steering Committee Worldwide variation in prevalence of symptoms of asthma, allergic rhinoconjunctivitis, and atopic eczema: ISAAC. Lancet 1998;351(9111):1225-32.

29. Konishi $\mathrm{H}$. A manual for the diagnoses of congenital anomalies (Gaihyou kikei sindan-no tebiki). Kanagawa, Japan: the study group on congenital anomalies in Kanagawa; 1981 (in Japanese).

30. Nishima S, Chisaka H, Fujiwara T, Furusho K, Hayashi S, Hiraba $\mathrm{K}$, et al. Surveys on the prevalence of pediatric bronchial asthma in Japan: a comparison between the 1982, 1992, and 2002 surveys conducted in the same region using the same methodology. Allergol Int. 2009;58(1):37-53. 
31. Okada E, Sasaki S, Saijo Y, Washino N, Miyashita C, Kobayashi $\mathrm{S}$, et al. Prenatal exposure to perfluorinated chemicals and relationship with allergies and infectious diseases in infants. Environ Res. 2012;112:118-25.

32. Yamada T, Morikawa M, Yamada T, Kishi R, Sengoku K, Endo $\mathrm{T}$, et al. First-trimester serum folate levels and subsequent risk of abortion and preterm birth among Japanese women with singleton pregnancies. Arch Gynecol Obstet. 2013;287(1):9-14.

33. Iida T, Todaka T. Measurement of dioxins in human blood: improvement of analytical method. Ind Health. 2003;41(3): 197-204.

34. Todaka T, Hirakawa H, Kajiwara J, Hori T, Tobiishi K, Onozuka $\mathrm{D}$, et al. Concentrations of polychlorinated dibenzo-p-dioxins, polychlorinated dibenzofurans, and dioxin-like polychlorinated biphenyls in blood collected from 195 pregnant women in Sapporo City, Japan. Chemosphere. 2007;69(8):1228-37.

35. Todaka T, Hirakawa H, Kajiwara J, Onozuka D, Sasaki S, Miyashita $\mathrm{C}$, et al. Concentrations of polychlorinated dibenzo-pdioxins, polychlorinated dibenzofurans, and polychlorinated biphenyls in blood and breast milk collected from pregnant women in Sapporo City, Japan. Chemosphere. 2011;85(11): 1694-700.

36. Todaka T, Hirakawa H, Tobiihi K, Iida T. New protocol of dioxins analysis in human blood. Fukuoka Igaku Zasshi. 2003;94(5):148-57.

37. Todaka T, Hori T, Hirakawa H, Kajiwara J, Yasutake D, Onozuka $\mathrm{D}$, et al. Congener-specific analysis of non-dioxin-like polychlorinated biphenyls in blood collected from 195 pregnant women in Sapporo City, Japan. Chemosphere. 2008;73(6):923-31.

38. Van den Berg M, Birnbaum LS, Denison M, De Vito M, Farland W, Feeley M, et al. The 2005 World Health Organization reevaluation of human and Mammalian toxic equivalency factors for dioxins and dioxin-like compounds. Toxicol Sci. 2006;93(2): $223-41$.

39. Inoue $\mathrm{K}$, Okada F, Ito $R$, Kato $S$, Sasaki $S$, Nakajima $S$, et al. Perfluorooctane sulfonate (PFOS) and related perfluorinated compounds in human maternal and cord blood samples: assessment of PFOS exposure in a susceptible population during pregnancy. Environ Health Perspect. 2004;112(11):1204-7.

40. Inoue K, Okada F, Ito R, Kawaguchi M, Okanouchi N, Nakazawa $\mathrm{H}$. Determination of perfluorooctane sulfonate, perfluorooctanoate and perfluorooctane sulfonamide in human plasma by column-switching liquid chromatography-electrospray mass spectrometry coupled with solid-phase extraction. J Chromatogr B Anal Technol Biomed Life Sci. 2004;810(1):49-56.

41. Kashino I, Okada E, Matsuura H. Development of UPLC-MS/MS for the determination of eleven PFC in human plasma. In: Proceedings of Japan Society for Environmental Chemistry, Ehime, Japan, 11-13 July, 2012.

42. Kanazawa A, Miyasita C, Okada E, Kobayashi S, Washino N, Sasaki S, et al. Blood persistent organochlorine pesticides in pregnant women in relation to physical and environmental variables in The Hokkaido Study on Environment and Children's Health. Sci Total Environ. 2012;426:73-82.

43. Konishi K, Sasaki S, Kato S. Effect of prenatal exposure to dioxins on birth weight. In: Proceedings of Persistent Organic Pollutants (POPs) Research in Asia, 2008, pp 362-67.

44. Konishi K, Sasaki S, Kato S, Ban S, Washino N, Kajiwara J, et al. Effects of prenatal exposure to dioxins and methyl mercury on birth weight. Organohalogen Compd. 2007;69:2109-11.

45. Ito Y, Yokota H, Wang R, Yamanoshita O, Ichihara G, Wang H, et al. Species differences in the metabolism of di(2-ethylhexyl) phthalate (DEHP) in several organs of mice, rats, and marmosets. Arch Toxicol. 2005;79(3):147-54.

46. Tsuboi T, Kawai T, Araki A, Kishi R. Determination of human urinary metabolites of five phthalate by GC/MS - study of elementally school children in Sapporo, Japan. In: Proceedings of International Society of Exposure Science, Seattle, USA, 2012, p. 40.

47. Araki A, Kawai T, Tsuboi T, Ait Bamai Y, Takeda T, Yoshioka $\mathrm{E}$, et al. Determination of phthalate metabolites in urine of children and their family-exposure assessment to plasticizer and flame retardants and their risk on children. In: Proceedings of International Society of Exposure Science, Seattle, USA, 2012, p. 72.

48. Yamamoto J, Hayama S, Sato N, Matsumura T, Kishi R, Sasaki $\mathrm{S}$, et al. Analysis of bisphenol A in biological sample. In: Proceedings of 20th Symposium on Environmental Chemistry, Kumamoto, Japan, 2011, pp 610-11.

49. Sasaki S, Braimoh TS, Yila TA, Yoshioka E, Kishi R. Selfreported tobacco smoke exposure and plasma cotinine levels during pregnancy - a validation study in Northern Japan. Sci Total Environ. 2011;412-413:114-8.

50. Kashima H, Kato M, Handa T. Neuropsychological investigation of the frontal function in chronic schizophrenia: results of a modified Wisconsin card sorting test. Jpn J Clin Psychiatry. 1985;14:1479-89 (in Japanese).

51. Kashima H, Kato M. Wisconsin card sorting test (Keio Version) (KWCST). Brain Sci Ment Disord. 1995;6:209-16 (in Japanese).

52. Sasaki S. Nihongoban yoshokisei yakuwari kodo shakudo (JPSAI) no sakusei: pre-School Activities Inventory no hoyaku. Stud Sociol Psychol Educ Inq Hum Soc. 2006;62:174-6.

53. Anme T, Shimada C, Katayama H. Evaluation of environmental stimulation for 18 months and the related factors. Nihon Koshu Eisei Zasshi. 1997;44(5):346-52.

54. Shiokawa H. Development of the life event questionnaire for parents: its use and reliability data. Jichi Med Univ J. 2007;30:165-72.

55. Kobayashi S, Sata F, Sasaki S, Ban S, Miyashita C, Okada E, et al. Genetic association of aromatic hydrocarbon receptor (AHR) and cytochrome P450, family 1, subfamily A, polypeptide 1 (CYP1A1) polymorphisms with dioxin blood concentrations among pregnant Japanese women. Toxicol Lett. 2013;219(3): 269-78.

56. Sasaki S, Kondo T, Sata F, Saijo Y, Katoh S, Nakajima S, et al. Maternal smoking during pregnancy and genetic polymorphisms in the Ah receptor, CYP1A1 and GSTM1 affect infant birth size in Japanese subjects. Mol Hum Reprod. 2006;12(2):77-83.

57. Sasaki S, Sata F, Katoh S, Saijo Y, Nakajima S, Washino N, et al. Adverse birth outcomes associated with maternal smoking and polymorphisms in the N-Nitrosamine-metabolizing enzyme genes NQO1 and CYP2E1. Am J Epidemiol. 2008;167(6): 719-26.

58. Yila TA, Sasaki S, Miyashita C, Braimoh TS, Kashino I, Kobayashi $\mathrm{S}$, et al. Effects of maternal 5,10-methylenetetrahydrofolate reductase C677T and A1298C Polymorphisms and tobacco smoking on infant birth weight in a Japanese population. J Epidemiol. 2012;22(2):91-102.

59. Konishi K, Sasaki S, Kato S, Ban S, Washino N, Kajiwara J, et al. Prenatal exposure to PCDDs/PCDFs and dioxin-like PCBs in relation to birth weight. Environ Res. 2009;109(7):906-13.

60. Nakajima S, Saijo Y, Kato S, Sasaki S, Uno A, Kanagami N, et al. Effects of prenatal exposure to polychlorinated biphenyls and dioxins on mental and motor development in Japanese children at 6 months of age. Environ Health Perspect. 2006;114(5): $773-8$.

61. Washino N, Saijo Y, Konishi K. The effect of prenatal exposure to dioxins on cord serum IGE. In: Proceedings of the 27th International Symposium on Halogenated Persistent Organic Pollutants, Tokyo, Japan, 2-7 September, 2007

62. Miyashita C, Sasaki S, Saijo Y, Washino N, Okada E, Kobayashi $\mathrm{S}$, et al. Effects of prenatal exposure to dioxin-like compounds on 
allergies and infections during infancy. Environ Res. 2011;111(4):551-8.

63. Washino N, Saijo Y, Sasaki S, Kato S, Ban S, Konishi K, et al. Correlations between prenatal exposure to perfluorinated chemicals and reduced fetal growth. Environ Health Perspect. 2009;117(4):660-7.

64. Okada E, Kashino I, Matsuura H, Sasaki S, Miyashita C, Yamamoto $\mathrm{J}$, et al. Temporal trends of perfluoroalkyl acids in plasma samples of pregnant women in Hokkaido, Japan, 2003-2011. Environ Int. 2013 (in press).

65. Okada E, Kashino I, Sasaki S, Miyashita C, Ikeno T, Araki A, et al. Relationship between prenatal exposure to perfluorinated compounds and allergic diseases in infants. In: Proceedings of International Society for Environmental Epidemiology, Basel, Switzerland, August, 2013

66. Rylander L, Stromberg U, Hagmar L. Dietary intake of fish contaminated with persistent organochlorine compounds in relation to low birthweight. Scand J Work Environ Health. 1996;22(4):260-6.

67. Baibergenova A, Kudyakov R, Zdeb M, Carpenter DO. Low birth weight and residential proximity to PCB-contaminated waste sites. Environ Health Perspect. 2003;111(10):1352-7.

68. Sonneborn D, Park HY, Petrik J, Kocan A, Palkovicova L, Trnovec T, et al. Prenatal polychlorinated biphenyl exposures in eastern Slovakia modify effects of social factors on birthweight. Paediatr Perinat Epidemiol. 2008;22(3):202-13.

69. Hertz-Picciotto I, Charles MJ, James RA, Keller JA, Willman E, Teplin S. In utero polychlorinated biphenyl exposures in relation to fetal and early childhood growth. Epidemiology. 2005;16(5): 648-56.

70. Vartiainen T, Jaakkola JJ, Saarikoski S, Tuomisto J. Birth weight and sex of children and the correlation to the body burden of PCDDs/PCDFs and PCBs of the mother. Environ Health Perspect. 1998;106(2):61-6.

71. Guo YL, Lambert GH, Hsu CC, Hsu MM. Yucheng: health effects of prenatal exposure to polychlorinated biphenyls and dibenzofurans. Int Arch Occup Environ Health. 2004;77(3):153-8.

72. Masuda Y. Fate of PCDF/PCB congeners and change of clinical symptoms in patients with Yusho PCB poisoning for 30 years. Chemosphere. 2001;43(4-7):925-30.

73. Wang SL, Lin CY, Guo YLL, Lin LY, Chou WL, Chang LW. Infant exposure to polychlorinated dibenzo-p-dioxins, dibenzofurans and biphenyls (PCDD/Fs, PCBs) - correlation between prenatal and postnatal exposure. Chemosphere. 2004;54(10):1459-73.

74. Suzuki G, Nakano M, Nakano S. Distribution of PCDDs/PCDFs and Co-PCBs in human maternal blood, cord blood, placenta, milk, and adipose tissue: dioxins showing high toxic equivalency factor accumulate in the placenta. Biosci Biotechnol Biochem. 2005;69(10): 1836-47.

75. Fletcher T, Steenland K, Savitz D. 2009. Status Report: PFOA and Immune Biomarkers in Adults Exposed to PFOA in Drinking Water in the Mid Ohio Valley: [http://www.c8sciencepanel.org/ pdfs/Status_Report_C8_and_Immune_markers_March2009.pdf] (Accessed 27 Sept 2011).

76. Harada K, Koizumi A, Saito N, Inoue K, Yoshinaga T, Date C, et al. Historical and geographical aspects of the increasing perfluorooctanoate and perfluorooctane sulfonate contamination in human serum in Japan. Chemosphere. 2007;66(2):293-301.

77. Jensen AA, Leffers H. Emerging endocrine disrupters: perfluoroalkylated substances. Int J Androl. 2008;31(2):161-9.

78. Kannan K, Corsolini S, Falandysz J, Fillmann G, Kumar KS, Loganathan BG, et al. Perfluorooctanesulfonate and related fluorochemicals in human blood from several countries. Environ Sci Technol. 2004;38(17):4489-95.
79. Yoshioka W, Peterson RE, Tohyama C. Molecular targets that link dioxin exposure to toxicity phenotypes. J Steroid Biochem Mol Biol. 2011;127(1-2):96-101.

80. Tsuchiya Y, Nakajima M, Yokoi T. Cytochrome P450-mediated metabolism of estrogens and its regulation in human. Cancer Lett. 2005;227(2):115-24.

81. Sørensen M, Autrup H, Møller P, Hertel O, Jensen SS, Vinzents $\mathrm{P}$, et al. Linking exposure to environmental pollutants with biological effects. Mutat Res Rev Mutat Res. 2003;544(2-3): 255-71.

82. Braimoh TS. Effects of maternal secondhand smoke exposure and gene polymorphisms of CYP1A1, EPHX1 and NAT2 on infant birth size. Doctoral thesis. Japan: Hokkaido University; 2012.

83. Damstra T, Barlow S, Bergman A, Kavlock R, Kraak Glen V. Global assessment of the state-of the science of endocrine disruptors. Geneva: World Health Organization; 2002.

84. Aschim EL, Nordenskjold A, Giwercman A, Lundin KB, Ruhayel Y, Haugen TB, et al. Linkage between cryptorchidism, hypospadias, and GGN repeat length in the androgen receptor gene. J Clin Endocrinol Metab. 2004;89(10):5105-9.

85. Thai HT, Kalbasi M, Lagerstedt K, Frisen L, Kockum I, Nordenskjold A. The valine allele of the V89L polymorphism in the 5 -alpha-reductase gene confers a reduced risk for hypospadias. J Clin Endocrinol Metab. 2005;90(12):6695-8.

86. Radpour R, Rezaee M, Tavasoly A, Solati S, Saleki A. Association of long polyglycine tracts (GGN repeats) in exon 1 of the androgen receptor gene with cryptorchidism and penile hypospadias in Iranian patients. J Androl. 2007;28(1):164-9.

87. Lichtenstein P, Carlstrom E, Rastam M, Gillberg C, Anckarsater $\mathrm{H}$. The genetics of autism spectrum disorders and related neuropsychiatric disorders in childhood. Am J Psychiatry. 2010;167(11):1357-63.

88. Ikeno T, Kobayashi S, Baba T, Kishi R. Literature review of the prevalence of attention-deficit hyperactivity disorder (ADHD) and its relation to environmental factors (ADHD no yuubyouritsu to youiku kankyou ni kansuru bunken review). Hokkaido J Public Health. 2011;25(2):53-9 (in Japanese).

89. Strang-Karlsson S, Raikkonen K, Pesonen AK, Kajantie E, Paavonen EJ, Lahti J, et al. Very low birth weight and behavioral symptoms of attention deficit hyperactivity disorder in young adulthood: The Helsinki study of very-low-birth-weight adults. Am J Psychiatry. 2008;165(10):1345-53.

90. Wolke D, Samara M, Bracewell M, Marlow N, Group EPS. Specific language difficulties and school achievement in children born at 25 weeks of gestation or less. J Pediatr. 2008;152(2): 256-62.

91. Hille ETM, den Ouden AL, Saigal S, Wolke D, Lambert M, Whitaker A, et al. Behavioural problems in children who weigh $1000 \mathrm{~g}$ or less at birth in four countries. Lancet. 2001;357(9269): 1641-3.

92. Hack M, Youngstrom EA, Cartar L, Schluchter M, Taylor HG, Flannery D, et al. Behavioral outcomes and evidence of psychopathology among very low birth weight infants at age 20 years. Pediatrics. 2004;114(4):932-40.

93. Hackett JA, Surani MA. DNA methylation dynamics during the mammalian life cycle. Philos Trans R Soc Lond B Biol Sci. 2013;368(1609):20110328.

94. Azumi K, Kobayashi S, Kishi R. Review: epigenetic research for the elucidation of the transgenelational effects of the environmental chemicals (Kankyou-kagaku bussitu no jisedaieikyou no kaimei ni okeru epigenetics kenkyuu). Hokkaido J Public Health. 2012;26(2):26-38 (in Japanese).

95. Baccarelli A, Bollati V. Epigenetics and environmental chemicals. Curr Opin Pediatr. 2009;21(2):243-51. 
96. Bollati V, Baccarelli A. Environmental epigenetics. Heredity (Edinb). 2010;105(1):105-12.

97. Murphy SK, Adigun A, Huang Z, Overcash F, Wang F, Jirtle RL, et al. Gender-specific methylation differences in relation to prenatal exposure to cigarette smoke. Gene. 2012;494(1):36-43.

98. Hoyo C, Fortner K, Murtha AP, Schildkraut JM, Soubry A, Demark-Wahnefried W, et al. Association of cord blood methylation fractions at imprinted insulin-like growth factor 2 (IGF2), plasma IGF2, and birth weight. Cancer Causes Control. 2012;23(4):635-45.

99. Vrijheid M, Casas M, Bergstrom A, Carmichael A, Cordier S, Eggesbo M, et al. European birth cohorts for environmental health research. Environ Health Perspect. 2012;120(1):29-37. 